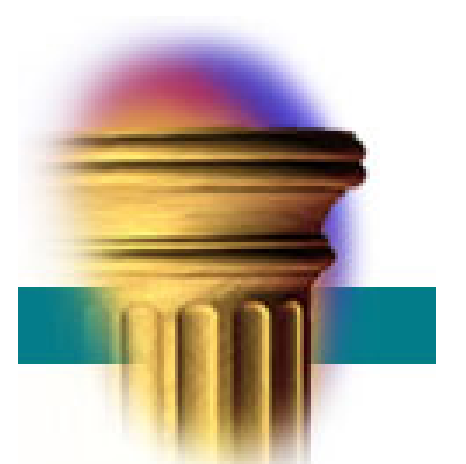

\title{
$\bigcirc$ \\ రृ \\ Computing Equilibria of Stochastic Heterogeneous Agent Models Using Decision Rule Histories
}

U

U

4

$\underline{1}$

m

Marcelo Veracierto

February, 2020

WP 2020-05

https://doi.org/10.21033/wp-2020-05

${ }^{*}$ Working papers are not edited, and all opinions and errors are the responsibility of the author(s). The views expressed do not necessarily reflect the views of the Federal Reserve Bank of Chicago or the Federal Reserve System. 


\title{
Computing Equilibria of Stochastic Heterogeneous Agent Models Using Decision Rule Histories
}

\author{
Marcelo Veracierto* \\ Federal Reserve Bank of Chicago, U.S.A
}

February, 2020

\begin{abstract}
This paper introduces a general method for computing equilibria with heterogeneous agents and aggregate shocks that is particularly suitable for economies with private information. Instead of the cross-sectional distribution of agents across individual states, the method uses as a state variable a vector of spline coefficients describing a long history of past individual decision rules. Applying the computational method to a Mirrlees RBC economy with known analytical solution recovers the solution perfectly well. This test provides considerable confidence on the accuracy of the method.
\end{abstract}

Keywords: Computational methods, heterogeneous agents, business cycles, private information.

\section{Introduction}

This paper introduces a general method for computing recursive equilibria of economies with both idiosyncratic and aggregate shocks. While the method is quite general and can be applied to a large class of models in which individual decision rules can be well approximated by spline

\footnotetext{
*I thank participants at various seminars and conferences for useful comments. The views expressed here do not necessarily reflect those of the Federal Reserve Bank of Chicago or the Federal Reserve System. Address: Federal Reserve Bank of Chicago, Research Department, 230 South LaSalle Street, Chicago, IL 60604. E-mail: mveracie@frbchi.org. Phone: (312) 322-5695.
} 
functions, it is particularly useful for computing equilibria of economies with private information. Economies with private information are difficult to solve because promised values are contingent on the realization of the aggregate shocks. This makes one of the endogenous state variables, the distribution of agents across promised values, not only infinite dimensional but state-contingent. Contrary to existing alternatives, the computational method described in this paper can handle this case without difficulty. Additionally, it has three features that make it attractive as a general computational method: 1) it keeps track of the full distribution of agents across individual states, 2) it can handle irregular shapes for this distribution, and 3) it incorporates the distribution's exact law of motion.

My basic strategy for the computational method is to parametrize individual decision rules as spline approximations and to keep long histories of the spline coefficients as state variables. Starting from the deterministic steady-state distribution, I use the history of decision rules implied by the spline coefficients to obtain the current distribution of agents across individual states. I do this by performing Monte Carlo simulations on a large panel of agents. All individual first-order conditions and aggregate feasibility constraints are then linearized with respect to the history of spline coefficients. The resulting linear model is then solved using standard methods. I show that a simple transformation can be applied to this solution in order to handle the case of contingent endogenous state variables.

After describing the computational method, I use it to solve the mechanism design problem of a Mirrlees economy with aggregate shocks. The economy, which belongs to the class considered in Veracierto (2019), is populated by agents that value consumption and leisure using logarithmic utility functions and which are subject to idiosyncratic shocks to their value of leisure. These shocks take only two possible values, are i.i.d. over time and across individuals, and are private information. Output, which can be consumed or invested, is produced using capital and labor as inputs to a Cobb-Douglas production function subject to aggregate productivity shocks. The aggregate shocks follow a standard $\mathrm{AR}(1)$ process.

A social planner designs dynamic contracts for the agents in this Mirrlees real business cycle (RBC) economy. Following the literature, a dynamic contract is given a standard recursive formulation where a promised value to the agent describes its state. Given the current state, the contract specifies current consumption, current hours worked, and next-period state-contingent promised values as a function of the value of leisure reported by the agent. Since the model has a 
large number of agents and the shocks to the value of leisure are idiosyncratic, the social planner needs to keep track of the whole distribution of promised values across individuals as a state variable. Given this distribution, the aggregate stock of capital, and the aggregate productivity level, the social planner seeks to maximize the present discounted utility of agents subject to incentive compatibility, promise keeping, and aggregate resource feasibility constraints.

Solving this mechanism design problem not only illustrates the applicability of the computational method to a problem that other methods cannot handle, but provides a strong test for it. The reason is that in Veracierto (2019) I provide a sharp analytical characterization of the solution to this mechanism design problem. In particular, I characterize the cyclical behavior of the consumption and leisure allocation rules across promised values, as well as the optimal amount of cross-sectional inequality in consumption and leisure over the business cycle. I also provide an analytical characterization for the optimal cyclical behavior of all macroeconomic variables in the economy. I find that the computational method passes this test extremely well: It recovers all the analytical results exactly. Since nothing in the computational method exploits the functional forms or structure of the Mirrlees RBC economy considered, this provides significant evidence about its accuracy. This finding indicates that the method should prove useful in a variety of other settings.

The paper is closely related to a vast literature on computational methods, but it has salient differences. ${ }^{2}$ The seminal papers by Krusell et al. (1998) and Den Haan (1996) summarize the cross-sectional distribution with a small set of moments. In contrast, the method in this paper keeps track of the whole distribution. Den Haan (1997) and Algan et al. (2008) also keep track of the whole distribution but parametrize the distribution with a flexible exponential polynomial form, allowing them to solve the model using quadrature and projection techniques. For many applications this may be an accurate and convenient approach, but for economies with odd-shaped distributions, it may not be. ${ }^{3}$ The method in this paper is able to handle odd shapes for the cross-

\footnotetext{
${ }^{2}$ See Algan et al. (2014) for a survey of computational methods.

${ }^{3}$ For instance, Achdou et al. (2017) have shown that in the Krusell et al. (1998) model with discrete income shocks (which has been widely used in the literature), the cross-sectional wealth distribution has spikes not only at the borrowing constraint, but in the interior of the state space. These types of distributions are generally difficult to describe with a flexible exponential form.
} 
sectional distribution as long as it is generated by smooth individual decision rules.

In addition to projection methods, the literature has explored perturbation methods, which are essentially local approximation methods around a deterministic steady state. Early versions include Campbell (1998), Dotsey et al. (1999), and Veracierto (2002) - the last two in the context of $(\mathrm{S}, \mathrm{s})$ economies. ${ }^{4}$ Perhaps the most widely known perturbation method is Reiter (2009), which is closely related to Campbell (1998). ${ }^{5}$ Instead of parametrizing the cross-sectional distribution as a polynomial, Reiter (2009) keeps a finite histogram of the distribution as a state variable. While the perturbation method allows him to greatly reduce the coarseness of the histogram, a limitation of Reiter's method is that the law of motion for the distribution needs to be approximated, and this can be a highly non-linear mapping. ${ }^{6}$ Instead, my method here embodies the exact law of motion for the distribution. Winberry (2018) introduces a very interesting perturbation method which, similarly to Algan et al. (2008), parametrizes the distribution with a flexible exponential polynomial form. The perturbation method allows him to carry a polynomial of large order as a state variable (or, equivalently, a large number of moments), which greatly improves the description of the distribution. However, his method also relies on an approximation for the law of motion of the cross-sectional distribution. Another powerful method has been introduced by Boppart et al.

\footnotetext{
${ }^{4}$ The method in this paper is actually a generalization of the approach used in Veracierto (2002).

${ }^{5}$ The recent method in Ahn et al. (2018) is an adaptation of Reiter's method to continuous time. Other perturbation methods in the literature include Preston and Roca (2007) and Mertens and Judd (2018), both of which perturb a deterministic steady state with no aggregate or idiosyncratic shocks. In contrast, the method in this paper perturbs a deterministic steady state with no aggregate shocks but positive idiosyncratic uncertainty.

${ }^{6}$ For instance, consider the Krusell et al. (1998) model. As has already been mentioned, in this model there is generally a mass of agents with the lowest idiosyncratic income level and zero assets (these agents are at the borrowing constraint). Now consider the steady state assets level chosen by these agents when they transit to a higher idiosyncratic income level. Suppose that this assets level falls within the first range of the histogram. Whenever there is a positive aggregate productivity shock, this choice of assets will generally increase. If the aggregate shock happens to be small enough that the modified assets level still falls within the first range of the histogram, there will be no effects at all on the histogram. However, if the shock is large enough that the modified assets level falls within the second range of the histogram, there will be a discrete reduction in the size of the first bar of the histogram and a discrete increase in the size of the second bar. Thus, the histogram bars change quite non-linearly with respect to the assets level chosen. This non-linearity problem can only be exacerbated when reducing the coarseness of the histogram.
} 
(2018). This method requires computing transitionary dynamics after an unexpected aggregate shock, starting from a given deterministic steady state. In many contexts this can readily be done. However, in economies with private information it can't. The reason is that since the distribution of agents across individual states is state-contingent, when the shock hits the economy the distribution shifts endogenously. As a consequence, there is no fixed starting point from which to start the deterministic transitionary dynamics. In fact, not only Boppart et al. (2018) have difficulties handling state-contingent distributions: none of the other papers cited above have addressed this case. On the contrary, the method in this paper can handle state-contingent distributions perfectly well.

The paper is organized as follows. Section 2 presents the computational method. Section 3 discusses its general applicability. Section 4 tests the method using a Mirrlees RBC economy with known analytical solution. Finally, Section 5 concludes the paper. All proofs are provided in an accompanying Technical Appendix.

\section{The computational method}

This section describes a general method for computing stationary equilibria of economies with heterogeneous agents and aggregate shocks. Although the method will be applied later on to an economy with asymmetric information, it is described here in general terms to make it applicable to a wide variety of settings.

The basic framework is as follows. The economy is populated by individual decision makers that solve maximization problems of the following form at every time period $t:^{7}$

$$
\begin{aligned}
& v_{h t}\left(a, x_{1}, x_{2}\right)=\max _{u_{h 1, t+1}, u_{h 2 t}}\left\{E_{t}\left[\sum_{s} R_{h}\left(s, a, x_{1}, x_{2},\left[u_{h 1, t+1}\left(s, a^{\prime}\right)\right]_{a^{\prime}},\left[u_{h 2 t}\left(s, a^{\prime}\right)\right]_{a^{\prime}}, z_{t}, p_{t}, p_{t+1}\right) \psi_{s}\right]+\right. \\
& \left.E_{t}\left[\sum_{s} \sum_{a^{\prime}} \beta_{h}\left(a, a^{\prime}, z_{t}, p_{t}, p_{t+1}\right) v_{h, t+1}\left(a^{\prime}, x_{1}^{\prime}\left(s, a^{\prime}\right), x_{2}^{\prime}\left(s, a^{\prime}\right)\right) \pi_{h}\left[a, a^{\prime}, u_{h 1, t+1}\left(s, a^{\prime}\right), u_{2 h t}\left(s, a^{\prime}\right)\right] \psi_{s}\right]\right\}
\end{aligned}
$$

\footnotetext{
${ }^{7}$ In what follows I use the convention that a variable is dated $t$ if its value becomes known when the date- $t$ aggregate shocks realize. If the dating of a variable $x$ is clear from the context, I avoid dating it explicitly and its next period value will be denoted by $x^{\prime}$. In particular, I avoid dating the arguments of individual value functions and decision rules.
} 
subject to

$$
\begin{aligned}
x_{1}^{\prime}\left(s, a^{\prime}\right) & =G_{h 1}\left(a, x_{1}, x_{2}, s, a^{\prime}, u_{h 1, t+1}\left(s, a^{\prime}\right)\right), \\
x_{2}^{\prime}\left(s, a^{\prime}\right) & =G_{h 2}\left(a, x_{1}, x_{2}, s, a^{\prime}, u_{h 2 t}\left(s, a^{\prime}\right)\right), \\
0 & \leq E_{t}\left[C_{h}\left(a, x_{1}, x_{2},\left[u_{h 1, t+1}\left(s, a^{\prime}\right)\right]_{s, a^{\prime}},\left[u_{h 2 t}\left(s, a^{\prime}\right)\right]_{s, a^{\prime}}, z_{t}, p_{t}, p_{t+1}\right)\right],
\end{aligned}
$$

where $h$ is the permanent type of the individual (e.g., being a household or a firm), $a$ is a vector of individual states that take a finite number of values (e.g., persistent idiosyncratic shocks), $z_{t}$ is a vector of aggregate shocks, $x_{1}$ is a vector of individual state variables whose values are contingent on the realizations of $a$ and $z_{t}, x_{2}$ is a vector of individual state variables whose values are contingent on the realization of $a$ but independent of $z_{t}, s$ is a vector of i.i.d. idiosyncratic shocks with distribution $\psi, u_{h 1, t+1}$ is a vector of $\left(a^{\prime}, z_{t+1}\right)$-contingent decision variables, $u_{h 2 t}$ is a vector of $\left(a^{\prime}\right)$ contingent decision variables, $p_{t}$ is a vector of equilibrium prices (whose stochastic process is taken as given by the individual), $G_{h 1}$ and $G_{h 2}$ define the laws of motion for $x_{1}$ and $x_{2}$, respectively, $C_{h}$ is a vector valued function defining constraints on $u_{h 1, t+1}$ and $u_{h 2 t}, \beta_{h}$ is a function that describes the discounting of future payoffs (allowing for idiosyncratic and/or aggregate preference shocks, as well as discounting using market prices), and $\pi_{h}$ describes the transition probabilities for $a$ (potentially affected by the individual's decisions). ${ }^{8}$ While $a$ and $s$ take a finite number of values, all other variables take real values. ${ }^{9}$ The solution to this sequence of maximization problems is a stochastic process for $v_{h t}, u_{h 1, t+1}$, and $u_{h 2 t}$, which are all functions over $\left(a, x_{1}, x_{2}\right)$. The permanent type $h$ implicitly defines the space in which $\left(a, x_{1}, x_{2}\right)$ lie. ${ }^{10}$. There is a finite number of different permanent types in the economy.

The distribution of $h$-type agents across individual states $\left(a, x_{1}, x_{2}\right)$ at the beginning of period

${ }^{8}$ While the dependence of $u_{h 1, t+1}$ or $u_{h 2 t}$ on $a^{\prime}$ is not critical, the dependence of $u_{h 1, t+1}$ on $z_{t+1}$ is what distinguishes it from $u_{h 2 t}$. Any decision variable that is not contingent on $z_{t+1}$ is assumed to be included in $u_{h 2 t}$. The same assumptions apply to $x_{1}$ and $x_{2}$. The presence of individual state and decision variables that depend on the realization of the aggregate shocks plays a crucial role in economies with private information.

${ }^{9}$ The reason I introduce the i.i.d. shocks $s$ explicitly instead of subsuming them in the vector $a$ is because of the restrictions across realizations of $s$ that equation (2.4) allows for. These cross-restrictions play a crucial role in certain economies with private information (e.g. representing incentive compatibility constraints).

${ }^{10} \mathrm{I}$ avoid introducing a subscript $h$ for these variables in order to simplify notation. However, the context will always make clear the permanent type $h$ that they correspond to. 
$t$ is described by a measure $\mu_{h t}$. The law of motion for $\mu_{h t}$ is given by the following equation:

$$
\begin{aligned}
\mu_{h, t+1}\left(\left\{a^{\prime}\right\} \times \mathcal{X}_{1} \times \mathcal{X}_{2}\right)= & \phi_{h}\left(\left\{a^{\prime}\right\} \times \mathcal{X}_{1} \times \mathcal{X}_{2}\right) \\
& +\sum_{s}\left(\int_{\mathcal{B}} \pi_{h}\left[a, a^{\prime}, u_{h 1, t+1}\left(a, x_{1}, x_{2}, s, a^{\prime}\right), u_{h 2 t}\left(a, x_{1}, x_{2}, s, a^{\prime}\right)\right] d \mu_{h t}\right) \psi_{s},
\end{aligned}
$$

for every $a^{\prime}$ and Borel sets $\mathcal{X}_{1}$ and $\mathcal{X}_{2}$, where

$$
\begin{aligned}
\mathcal{B}= & \left\{\left(a, x_{1}, x_{2}\right): G_{h 1}\left(a, x_{1}, x_{2}, s, a^{\prime}, u_{h 1, t+1}\left(a, x_{1}, x_{2}, s, a^{\prime}\right)\right) \in \mathcal{X}_{1}\right. \\
& \text { and } \left.G_{h 2}\left(a, x_{1}, x_{2}, s, a^{\prime}, u_{h 2 t}\left(a, x_{1}, x_{2}, s, a^{\prime}\right)\right) \in \mathcal{X}_{2}\right\}
\end{aligned}
$$

The measure $\phi_{h}$ describes an exogenous endowment of new agents (e.g., to accommodate exogenous entry of firms in a firm dynamics context or newborns in a households life cycle context), while the second term describes the endogenous evolution of the distribution. Observe that since $u_{h 1, t+1}$ is contingent on the realization of $z_{t+1}$, the same is generally true for $\mu_{h, t+1}$. I assume that $\mu_{h 1}$, $\phi_{h}$, and $\pi_{h}$ are such that the total number of $h$-type agents $\mu_{h t}$ is constant over time and equal to $\Gamma_{h}$, independent of the stochastic process $\left\{u_{h 1, t+1}, u_{h 2 t}\right\}_{t=1}^{\infty}$.

In what follows, it will be useful to differentiate the $h$-type of agents that are infinitely lived and for which the maximization problem (2.1)-(2.4) is independent of $a$ and $s$. Henceforth, all variables corresponding to such "representative" types of agents will be denoted with a subscript $r$, while the $h$ subscript will be reserved for heterogeneous types. An important characteristic of representative types of agents is that the measure $\mu_{r t}$ describing their distribution across individual states will have mass at a single point $\left(x_{r 1 t}, x_{r 2, t-1}\right)$. Therefore, it will be convenient to replace $\mu_{r t}$ with that single point and replace the law of motion (2.5)-(2.6) with

$$
\begin{aligned}
x_{r 1, t+1} & =G_{r 1}\left(x_{r 1, t}, x_{r 2, t-1}, u_{r 1, t+1}\left(x_{r 1, t}, x_{r 2, t-1}\right)\right), \\
x_{r 2 t} & =G_{r 2}\left(x_{r 1 t}, x_{r 2, t-1}, u_{r 2 t}\left(x_{r 1, t}, x_{r 2, t-1}\right)\right) .
\end{aligned}
$$

The stochastic process for $p_{t}$, which is taken as given in the maximization problems (2.1)-(2.4), is an equilibrium process if for every $t$,

$Q\left(z_{t},\left[\sum_{s}\left(\int M_{h}\left(a, x_{1}, x_{2},\left[u_{h 2 t}\left(a, x_{1}, x_{2}, s, a^{\prime}\right)\right]_{a^{\prime}}\right) d \mu_{h t}\right) \psi_{s}\right]_{h},\left[x_{r 1 t}, x_{r 2, t-1}, u_{r 2 t}\left(x_{r 1 t}, x_{r 2, t-1}\right)\right]_{r}\right)=0$,

where $Q$ is a vector valued function (of the same dimensionality as $p_{t}$ ) describing aggregate feasibility and/or market clearing conditions, $M_{h}$ is a vector valued function that determines which 
moments of $\mu_{h t}$ are arguments of $Q$, and $\left(x_{r 1 t}, x_{r 2, t-1}, u_{r 2 t}\right)$ are the states and decision functions of the $r$-type of representative agents. Observe that the $z_{t+1}$-contingent decision variables $u_{h 1, t+1}$ and $u_{r 1, t+1}$ do not enter $Q$.

The vector of aggregate shocks $z_{t}$ follows an $\operatorname{AR}(1)$ process $z_{t+1}=N z_{t}+\varepsilon_{t+1}$, where $E_{t}\left[\varepsilon_{t+1}\right]=$ 0 .

The high dimensionality of the equilibrium objects makes computing stationary equilibria for this type of setting a nontrivial task. My approach will be to replace these objects with a finite set of numbers that approximate them arbitrarily well. Moreover, the finite representation will be chosen in such a way that the law of motion corresponding to equations (2.5)-(2.6) will be a linear mapping. All first-order conditions and aggregate feasibility constraints will then be linearized with respect to the variables in the finite representation (at their deterministic steady state values), delivering a linear rational expectations model that can be solved using almost standard methods. ${ }^{11}$ Since under the chosen finite representation the law of motion corresponding to equations (2.5)-(2.6) is already linear, this method has the advantage that the linearization does not introduce any further approximation errors to it: The method not only keeps track of the distributions $\mu_{h t}$ arbitrarily well over all of their supports, but also uses their exact laws of motion. Since the method performs a linearization at the deterministic steady state equilibrium values of all variables, it requires computing these values as a first step.

\subsection{Computing the deterministic steady state}

While computing a deterministic steady state for this type of model is standard, this section describes in detail a specific algorithm that serves to introduce objects and notation that will be needed later on. Throughout the section, I assume that a deterministic steady state equilibrium exists.

In order to compute a steady state, I start by making $z_{t}$ identical to zero and fixing the price vector at some value $p$. For each $r$-type of representative agent, the vector of time invariant state and decision variables $\left(x_{1 r}, x_{2 r}, u_{1 r}, u_{2 r}\right)$ can then be directly obtained from the first-order conditions of the corresponding maximization problem.

I find it convenient to solve the maximization problems given by equations (2.1)-(2.4) using

\footnotetext{
${ }^{11}$ The "almost" qualifier will be clarified later on.
} 
spline approximations and value function iterations. ${ }^{12}$ To start, I restrict each component of the vector of endogenous individual state variables $\left(x_{1}, x_{2}\right)$ for each $h$-type agent to lie in a closed interval and define a set of grid points in it that includes the extremes. ${ }^{13}$ The Cartesian product of all these sets of grid points defines a finite set of grid points for $\left(x_{1}, x_{2}\right)$, which is described by a vector $\left(\bar{x}_{1 j}, \bar{x}_{2 j}\right)_{j=1}^{J_{h}}$. Given the value function $v_{h}$ from the previous iteration, which is used to evaluate $\left(x_{1}^{\prime}, x_{2}^{\prime}\right)$ (possibly outside the grid points), the maximization problem in equations (2.1)-(2.4) is solved for only at the grid points $\left(\bar{x}_{1 j}, \bar{x}_{2 j}\right)_{j=1}^{J_{h}}$. Once, the vectors of new values $\bar{v}_{h}=\left[v_{h}\left(a, \bar{x}_{1 j}, \bar{x}_{2 j}\right)\right]_{a, j}, \bar{u}_{h 1}=\left[u_{h 1}\left(a, \bar{x}_{1 j}, \bar{x}_{2 j}, s, a^{\prime}\right)\right]_{a, j, s, a^{\prime}}$, and $\bar{u}_{h 2}=\left[u_{h 2}\left(a, \bar{x}_{1 j}, \bar{x}_{2 j}, s, a^{\prime}\right)\right]_{a, j, s, a^{\prime}}$ are obtained, I extend their values to the full domain of $\left(x_{1}, x_{2}\right)$ using splines. These value function iterations continue until $\bar{v}_{h}$ converges. Observe that the solution obtained depends on the price vector $p$, which has been fixed.

For heterogenous agents, the steady state version of equations (2.5)-(2.6) describes the recursion that the time invariant distribution $\mu_{h}$ has to satisfy. This equation corresponds to the case of a continuum of agents. However, I find it convenient to perform the recursion in the case of a large but finite number of agents. In particular, consider a large but finite number $I_{h}$ of $h$-type agents and endow them with some individual states $\left(a, x_{1}, x_{2}\right)$. Using the functions $u_{h 1}$ and $u_{h 2}$ already obtained, I simulate the evolution of the individual states of these $I_{h}$ agents for a large number of periods $T$. To be precise, if an $h$-type agent $i$ has the individual state $\left(a, x_{1}, x_{2}\right)$ at the beginning of the current period, then the individual state $\left(a^{\prime}, x_{1}^{\prime}, x_{2}^{\prime}\right)$ at the beginning of the following period is randomly determined as follows:

(i) with probability $\pi_{h}\left[a, a^{\prime}, u_{h 1}\left(a, x_{1}, x_{2}, s, a^{\prime}\right), u_{h 2}\left(a, x_{1}, x_{2}, s, a^{\prime}\right)\right] \psi_{s}$, it is given by

(ii) with probability $1-\sum_{s, a^{\prime}} \pi\left[a, a^{\prime}, u_{h 1}\left(a, x_{1}, x_{2}, s, a^{\prime}\right), u_{h 2}\left(a, x_{1}, x_{2}, s, a^{\prime}\right)\right] \psi_{s}$ it is determined by $\phi_{h}$.

Observe that the transition in (ii) takes place when the individual dies and is replaced by a

\footnotetext{
${ }^{12}$ For representative agents with state contingent state variables $x_{1 r}$, it will be important to follow the procedure described in this paragraph as well since the steady state objects described here will be needed later on.

${ }^{13}$ When restricting each of these variables to lie in a closed interval, one should modify the steady state maximization problem (2.1)-(2.4) to incorporate the corresponding constraints on $x_{1}^{\prime}$ and $x_{2}^{\prime}$. The use of splines is what requires each component of $\left(x_{1}, x_{2}\right)$ to lie in a closed interval.
} 
newborn whose initial state is unrelated to the state of the predecessor.

Simulating the $I_{h}$ agents and their descendants for $T$ periods using the law of motion in (2.10), I obtain a realized distribution $\left(a^{i}, x_{1}^{i}, x_{2}^{i}\right)_{i=1}^{I_{h}}$ of individual states across the $I_{h}$ agents. Doing this for every $h$-type, the aggregate feasibility conditions can then be computed as

$$
Q\left(0,\left[\sum_{s}\left(\Gamma_{h} \frac{1}{I_{h}} \sum_{i=1}^{I_{h}} M_{h}\left(a^{i}, x_{1}^{i}, x_{2}^{i},\left[u_{h 2}\left(a^{i}, x_{1}^{i}, x_{2}^{i}, s, a^{\prime}\right)\right]_{a^{\prime}}\right)\right) \psi_{s}\right]_{h},\left[x_{r 1}, x_{r 2}, u_{r 2}\right]_{r}\right)=0 .
$$

Observe that by the law of large numbers, equation (2.11) will become an arbitrarily good approximation of equation (2.9) as all $I_{h}$ and $T$ tend to infinity.

If equation (2.11) is not satisfied, the price vector $p$ must be changed until it is. This represents a standard root finding problem.

\subsection{Computing the stationary stochastic solution}

As I already mentioned, computing the stationary stochastic solution requires linearizing the first-order conditions to the maximization problems given by equations (2.1)-(2.4), the laws of motion (2.5)-(2.6), the laws of motion (2.7)-(2.8), and the aggregate feasibility conditions given by equation (2.9) with respect to a convenient set of variables.

In order to illustrate some of the issues involved in the linearization of the first-order conditions, I will use equation (2.1) as an example since it represents the most complex type. ${ }^{14}$ The first issue is the existence of a continuum of equations (2.1), since $\left(x_{1}, x_{2}\right)$ take a continuum of values. I solve this "curse of dimensionality" by considering the equation only at the grid points $\left(\bar{x}_{1 j}, \bar{x}_{2 j}\right)_{j=1}^{J_{h}}$ that were used in the computation of the deterministic steady state. Another issue is that each of this finite number of equations depends on the infinite dimensional object $v_{h, t+1}$, since it is a function of $\left(x_{1}^{\prime}, x_{2}^{\prime}\right)$, and I need to evaluate these variables outside the grid points. In this case, I solve the "curse of dimensionality" by considering that $v_{h, t+1}$ is a spline approximation and, therefore,

\footnotetext{
${ }^{14}$ Equation (2.1) enters the set of first order conditions if the transition probabilities $\pi_{h}$ depend on $u_{h 1, t+1}$ or $u_{h 2 t}$. In this case, the level of $v_{h t}$ enters the first order conditions and the definitional equation (2.1) must be included. If $\pi_{h}$ does not depend on $u_{h 1, t+1}$ or $u_{h 2 t}$, only the derivatives of $v_{h t}$ enter the first order conditions. However, the issues discussed here in the context of equation (2.1) apply to other first-order conditions, including the definitional equation for the derivatives of $v_{h t}$. For reasons I will explain in Section 2.3, it is important to write first order conditions using the derivatives of the value function and not as second order stochastic difference equations.
} 
is completely determined by the vector $\bar{v}_{h, t+1}=\left[v_{h, t+1}\left(a, \bar{x}_{1 j}, \bar{x}_{2 j}\right)\right]_{a, j}$, i.e., by the value of the function at the grid points. Consequently, after substituting equations (2.2)-(2.3) into equation (2.1) and linearizing at the corresponding steady state values, I am left with the following finite set of equations:

$$
0=E_{t}\left\{\mathcal{L}_{h}^{v}\left(\bar{v}_{h, t}, \bar{u}_{h 1, t+1}, \bar{u}_{h 2 t}, z_{t}, p_{t}, p_{t+1}, \bar{v}_{h, t+1}\right)\right\}
$$

where $\bar{u}_{h 1, t+1}=\left[u_{h 1, t+1}\left(a, \bar{x}_{1 j}, \bar{x}_{2 j}, s, a^{\prime}\right)\right]_{a, j, s, a^{\prime}}, \bar{u}_{h 2 t}=\left[u_{h 2 t}\left(a, \bar{x}_{1 j}, \bar{x}_{2 j}, s, a^{\prime}\right)\right]_{a, j, s, a^{\prime}}$ and $\mathcal{L}_{h}^{v}$ is a vector valued linear function with the same dimensionality as $\bar{v}_{h t}$.

Particular attention should be given to the first-order conditions corresponding to grid points $\left(a, \bar{x}_{1 j}, \bar{x}_{2 j}\right)$ for which the deterministic steady state choice of some component of $x_{1}^{\prime}\left(s, a^{\prime}\right)$ or $x_{2}^{\prime}\left(s, a^{\prime}\right)$ hits one of the extremes imposed by the use of spline approximations. At these grid points, the maximization problem (2.1)-(2.4) should be modified by imposing the constraint that the corresponding component of equation (2.2) or (2.3) must evaluate to the corresponding extreme. The first-order conditions used at these grid points should be those of the modified problem. A consequence of this is that if the optimal choice of some component of $x_{1}^{\prime}\left(s, a^{\prime}\right)$ or $x_{2}^{\prime}\left(s, a^{\prime}\right)$ hits an extreme in the steady state solution, it will always hit it in the stochastic solution. This will certainly distort the stochastic decision rules close to the extremes, so in practice one should choose these extremes far enough that the invariant distribution $\mu_{h}$ puts little mass close to them (minimizing the relevance of these distortions).

Linearizing the aggregate feasibility conditions described by equation (2.9) presents more complicated issues because of their dependence on the integrals $\left[\int M_{h} d \mu_{h t}\right]_{h}$. To make progress, these integrals must be represented with a convenient finite set of variables. To do this, I follow a strategy that is closely related to the one used in Section 2.1 for computing statistics under the invariant distributions. In particular, for each heterogenous type of agent $h$, consider the same large but finite number of agents $I_{h}$ used in that section and endow them with the same realized distribution of individual states $\left(a^{i}, x_{1}^{i}, x_{2}^{i}\right)_{i=1}^{I_{h}}$ that was obtained when computing the steady state. Now, assume that these agents populated the economy $N$ time periods ago and consider the history $\left\{u_{h 1, t+1-n}, u_{h 2, t-n}\right\}_{n=1}^{N}$ of decision rules that were realized during the last $N$ periods (where $t$ is considered to be the current period). Since these decision rules are spline approximations, this history can be represented by the finite list of values $\left\{\bar{u}_{h 1, t+1-n}, \bar{u}_{h 2, t-n}\right\}_{n=1}^{N}$. Using this history of decision rules, I can simulate the evolution of individual states for the $I_{h}$ agents and their descen- 
dants during the last $N$ time periods to update the distribution of individual states from the initial $\left(a^{i}, x_{1}^{i}, x_{2}^{i}\right)_{i=1}^{I_{h}}$ to a current distribution $\left(a_{t}^{i}, x_{1 t}^{i}, x_{2, t-1}^{i}\right)_{i=1}^{I_{h}}$. In particular, I can initialize the distribution of individual states at the beginning of period $t-N$ as $\left(a_{t-N}^{i}, x_{1, t-N}^{i}, x_{2, t-N-1}^{i}\right)=\left(a^{i}, x_{1}^{i}, x_{2}^{i}\right)$, for $i=1, \ldots, I_{h}$. Given a distribution of individual states $\left[\left(a_{t-n}^{i}, x_{1, t-n}^{i}, x_{2, t-n-1}^{i}\right)\right]_{i=1}^{I_{h}}$ at period $t-n$, the individual state $\left(a_{t-n+1}^{i}, x_{1, t-n+1}^{i}, x_{2, t-n}^{i}\right)$ of each agent $i$ at period $t-n+1$ is randomly determined as follows:

(i) with probability $\pi_{h}\left[a_{t-n}^{i}, a^{\prime}, u_{h 1, t+1-n}^{i}\left(s, a^{\prime}\right), u_{h 2, t-n}^{i}\left(s, a^{\prime}\right)\right] \psi_{s}$, it is given by $\left(a^{\prime}, G_{h 1, t+1-n}^{i}\left(s, a^{\prime}\right)\right.$, $\left.G_{h 2, t-n}^{i}\left(s, a^{\prime}\right)\right)$, where $\left(u_{h 1, t+1-n}^{i}\left(s, a^{\prime}\right), u_{h 2, t-n}^{i}\left(s, a^{\prime}\right), G_{h 1, t+1-n}^{i}\left(s, a^{\prime}\right), G_{h 2, t-n}^{i}\left(a^{\prime}\right)\right)$ are the values of $\left(u_{h 1, t+1-n}, u_{h 2, t-n}, G_{h 1}, G_{h 2}\right)$ evaluated at $\left(a_{t-n}^{i}, a^{\prime}, x_{1, t-n}^{i}, x_{2, t-n-1}^{i}, s, a^{\prime}\right)$,

(ii) with probability $1-\sum_{s, a^{\prime}} \pi_{h}\left[a_{t-n}^{i}, a^{\prime}, u_{h 1, t+1-n}^{i}\left(s, a^{\prime}\right), u_{h 2, t-n}^{i}\left(s, a^{\prime}\right)\right] \psi_{s}$, it is determined by $\phi_{h}$.

Proceeding recursively for $n=N, N-1, \ldots, 1$, I obtain a realized distribution $\left(a_{t}^{i}, x_{1 t}^{i}, x_{2, t-1}^{i}\right)_{i=1}^{I_{h}}$ at the beginning of period $t$. This distribution can be used to compute statistics under the distribution $\mu_{h t}$. In particular, having followed the above procedure for each $h$-type of heterogeneous agents, I can rewrite equation $(2.9)$ as

$$
\begin{aligned}
& 0= Q\left(z_{t},\left[\sum_{s}\left(\Gamma_{h} \frac{1}{I_{h}} \sum_{i=1}^{I_{h}} M_{h}\left(a_{t}^{i}, x_{1 t}^{i}, x_{2, t-1}^{i},\left[u_{h 2 t}\left(a_{t}^{i}, x_{1 t}^{i}, x_{2, t-1}^{i}, s, a^{\prime}\right)\right]_{a^{\prime}}\right)\right) \psi_{s}\right]_{h},\right. \\
&\left.,\left[x_{r 1 t}, x_{r 2, t-1}, u_{r 2 t}\left(x_{r 1 t}, x_{r 2, t-1}\right)\right]_{r}\right)
\end{aligned}
$$

Since $u_{h 2 t}$ and $u_{r 2 t}$ are spline approximations, they can also be summarized by their values at the grid points $\bar{u}_{h 2 t}$ and $\bar{u}_{r 2 t} \cdot{ }^{15}$ As a consequence, equation (2.13) can be linearized at the deterministic steady state values to get the following finite set of equations:

$$
0=\mathcal{L}^{Q}\left(z_{t},\left[\left\{\bar{u}_{h 1, t+1-n}\right\}_{n=1}^{N},\left\{\bar{u}_{h 2, t-n}\right\}_{n=0}^{N}\right]_{h},\left[x_{r 1 t}, x_{r 2, t-1}, \bar{u}_{r 2 t}\right]_{r}\right)
$$

where $\mathcal{L}^{Q}$ is a vector valued linear function. ${ }^{16}$

\footnotetext{
${ }^{15}$ For simplicity, I assume here that all representative agents have state-contingent states $x_{1 r}$. However, for representative agents with no state-contingent states, instead of writing equation (2.13) in terms of their decision rules $u_{r 2 t}$, it is often more convenient to write it directly in terms of the values of their type-2 decision variables at date $t$. Consequently, for this type of representative agents, $\bar{u}_{r 2 t}$ in equations (2.14) and (2.18) is not a vector of spline coefficients but a vector of values for type- 2 decision variables.

${ }^{16}$ Taking numerical derivatives of equation (2.13) with respect to each spline coefficient in the list
} 
My approach of representing the distribution $\mu_{h t}$ with a finite history of values greatly simplifies the description of the law of motion in equations (2.5)-(2.6). In fact, updating the distribution $\mu_{h t}$ is merely reduced to updating those histories. In particular, the date- $(t+1)$ histories can be obtained from the date-t histories and the current values $\bar{u}_{h 1, t+1}$ and $\bar{u}_{h 2 t}$ using the following equations:

$$
\begin{aligned}
& \bar{u}_{h 1,(t+1)-n}=\bar{u}_{h 1, t-(n-1)} \\
& \bar{u}_{h 2,(t+1)-n}=\bar{u}_{h 2, t-(n-1)},
\end{aligned}
$$

for $n=1, \ldots, N$. Observe that the law of motion described by equations (2.15)-(2.16) is already linear, so no further linearization is needed. Also observe that the variables that are $N$ periods old in the date- $t$ history are dropped from the date- $(t+1)$ history. Thus, the law of motion described by these equations introduces a truncation. However, introducing a life cycle structure to the $h$-type of heterogenous agents will make the consequences of this truncation negligible. The reason is that the truncation only affects agents surviving for $N$ consecutive periods and, given sufficiently small survival probabilities and/or a sufficiently large $N$, there will be very few of these agents. Apart from this negligible truncation, there are no further approximations errors in the representation of the law of motion given by equations (2.5)-(2.6) - a crucial benefit of using the computational method described in this paper.

Since all $u_{r 1 t, t+1}$ and $u_{r 2 t}$ are also spline approximations they are summarized by their values at the grid points $\bar{u}_{r 1 t, t+1}$ and $\bar{u}_{r 2 t}$. The laws of motion (2.7)-(2.8) can then be linearized to obtain

$$
\begin{aligned}
0 & =\mathcal{L}^{G_{r 1}}\left(x_{r 1, t+1}, x_{r 1 t}, x_{r 2, t-1}, \bar{u}_{r 1 t, t+1}\right), \\
0 & =\mathcal{L}^{G_{r 2}}\left(x_{r 2 t}, x_{r 1 t}, x_{r 2, t-1}, \bar{u}_{r 2 t}\right)
\end{aligned}
$$

where $\mathcal{L}^{G_{r 1}}$ and $\mathcal{L}^{G_{r 2}}$ are vector valued linear functions of the same dimensionality as $x_{r 1, t+1}$ and $x_{r 2 t}$, respectively.

$\left[\left\{\bar{u}_{h 1, t+1-n}\right\}_{n=1}^{N},\left\{\bar{u}_{h 2, t-n}\right\}_{n=0}^{N}\right]_{h}$ requires simulating $I_{h}$ agents over $N$ periods. Thus, obtaining the linear function $\mathcal{L}^{Q}$ requires performing a large number of Monte Carlo simulations. Moreover, minimizing sampling errors requires a large value for $I_{h}$ (in practice I work with panels of about 10 million individuals). While this seems a daunting task, it is easily parallelizable. Thus, using massively parallel computer systems (such as GPU accelerators) can play an important role in reducing computing times and keeping the task manageable. 
Once all equations have been linearized, I am left with a stochastic linear rational expectations model with a non-standard feature - namely, that some of the decision variables during the current period and some of the endogenous states during the next period are contingent on the realization of the aggregate shocks during the next period. Fortunately, this difficulty can be handled easily. The reason is that the stochastic state-contingent solution that I seek can be easily constructed from the solution to the deterministic version of the model, and this version has a standard structure that can be solved using well known methods. In what follows, I describe the linear stochastic model in detail and show how to perform this transformation.

\subsection{Solving the linearized model}

Define the following vectors:

$$
\begin{aligned}
x_{t}^{1} & =\left[\left[\left\{\Delta \bar{u}_{h 1, t+1-n}\right\}_{n=1}^{N}\right]_{h},\left[\Delta x_{r 1 t}\right]_{r}\right], \\
x_{t-1}^{2} & =\left[\left[\left\{\Delta \bar{u}_{h 2, t-n}\right\}_{n=1}^{N}\right]_{h},\left[\Delta x_{r 2, t-1}\right]_{r}\right], \\
y_{t+1}^{1} & =\left[\left[\Delta \bar{u}_{h 1, t+1}\right]_{h},\left[\Delta \bar{u}_{r 1, t+1}\right]_{r}\right], \\
y_{t}^{2} & =\left[\left[\Delta \bar{v}_{h t}, \Delta\left(\frac{\partial \bar{v}_{h t}}{\partial x}\right), \Delta \bar{q}_{h t}, \Delta \bar{u}_{h 2 t}\right]_{h},\left[\Delta \bar{v}_{r t}, \Delta\left(\frac{\partial \bar{v}_{r t}}{\partial x}\right), \Delta \bar{q}_{r t}, \Delta \bar{u}_{r 2 t}\right]_{r}, \Delta p_{t}\right],
\end{aligned}
$$

where $\Delta$ represents deviations from steady state values. $\partial \bar{v}_{h t} / \partial x$ and $\bar{q}_{h t}$ are the derivatives of $v_{h t}$ and the Lagrange multipliers of constraints (2.4), respectively, evaluated at the grid points of the $h$-type of heterogeneous agents. $\partial \bar{v}_{r t} / \partial x$ and $\bar{q}_{r t}$ are similar objects but for the $r$-type of representative agents. The linearized model can then be written as

$$
\begin{aligned}
0= & B_{11} x_{t}^{1}+B_{12} x_{t-1}^{2}+C_{12} y_{t}^{2}+D_{1} z_{t}, \\
0= & A_{21} x_{t+1}^{1}+B_{21} x_{t}^{1}+B_{22} x_{t-1}^{2}+C_{21} y_{t+1}^{1}, \\
0= & A_{32} x_{t}^{2}+B_{31} x_{t}^{1}+B_{32} x_{t-1}^{2}+C_{32} y_{t}^{2}, \\
0= & H_{41} x_{t}^{1}+H_{42} x_{t-1}^{2}+J_{42} y_{t+1}^{2}+K_{41} y_{t+1}^{1}+K_{42} y_{t}^{2}+M_{4} z_{t}, \\
0= & E_{t}\left\{F_{52} x_{t+1}^{2}+G_{52} x_{t}^{2}+H_{51} x_{t}^{1}+H_{52} x_{t-1}^{2}+J_{52} y_{t+1}^{2}+K_{51} y_{t+1}^{1},\right. \\
& \left.+K_{52} y_{t}^{2}+L_{5} z_{t+1}+M_{5} z_{t}\right\} \\
z_{t+1}= & N z_{t}+\varepsilon_{t+1},
\end{aligned}
$$

where (2.23) represents the aggregate feasibility constraints (equation 2.14), (2.24) is the law of motion for $x_{t}^{1}$ (equations 2.15 and 2.17), (2.25) is the law of motion for $x_{t-1}^{2}$ (equations 2.16 and 
2.18), (2.26) is the first-order conditions for $u_{h 1, t+1}$ and $u_{r 1, t+1}$ evaluated at the grid points (which must hold almost surely), and (2.27) represents the constraints (2.4), the first-order conditions for $u_{h 2 t}$ and $u_{r 2 t}$, the definitions of $\bar{v}_{h t}$ and $\bar{v}_{r t}$ (e.g., equation 2.12), and the envelope conditions for $\partial \bar{v}_{h t} / \partial x$ and $\partial \bar{v}_{r t} / \partial x$, all evaluated at the grid points (these equations must all hold in expectation). ${ }^{17}$ I seek a recursive solution to equations (2.23)-(2.28) of the following form:

$$
\begin{aligned}
x_{t+1}^{1} & =\Omega_{11} x_{t}^{1}+\Omega_{12} x_{t-1}^{2}+\Psi_{1} z_{t}+\Theta_{1} z_{t+1}, \\
x_{t}^{2} & =\Omega_{21} x_{t}^{1}+\Omega_{22} x_{t-1}^{2}+\Psi_{2} z_{t}, \\
y_{t+1}^{1} & =\Phi_{11} x_{t}^{1}+\Phi_{12} x_{t-1}^{2}+\Gamma_{1} z_{t}+\Lambda_{1} z_{t+1}, \\
y_{t}^{2} & =\Phi_{21} x_{t}^{1}+\Phi_{22} x_{t-1}^{2}+\Gamma_{2} z_{t} .
\end{aligned}
$$

My strategy will be to construct it from the recursive solution to the deterministic version of equations (2.23)-(2.28), in which $\varepsilon_{t+1}$ is set to zero and the expectations operator is dropped. ${ }^{18}$ This deterministic version has identical structure as the system analyzed in Uhlig (1999) and can be solved using identical methods. ${ }^{19}$ Its solution has the following form:

$$
\begin{aligned}
x_{t+1}^{1} & =P_{11} x_{t}^{1}+P_{12} x_{t-1}^{2}+Q_{1} z_{t}, \\
x_{t}^{2} & =P_{21} x_{t}^{1}+P_{22} x_{t-1}^{2}+Q_{2} z_{t}, \\
y_{t+1}^{1} & =R_{11} x_{t}^{1}+R_{12} x_{t-1}^{2}+S_{1} z_{t}, \\
y_{t}^{2} & =R_{21} x_{t}^{1}+R_{22} x_{t-1}^{2}+S_{2} z_{t} .
\end{aligned}
$$

Proposition 1 Let (2.33)-(2.36) be the solution to the deterministic version of equations (2.23)(2.28). Define $\Omega_{11}=P_{11}, \Omega_{12}=P_{12}, \Omega_{21}=P_{21}, \Omega_{22}=P_{22}, \Psi_{2}=Q_{2}, \Phi_{11}=R_{11}, \Phi_{12}=R_{12}$,

\footnotetext{
${ }^{17}$ Actually, only the constraints in (2.4) that hold with equality are included in the system of equations. Also, only the Lagrange multipliers of these constraints are included in $\bar{q}_{h t}$ and $\bar{q}_{r t}$ in equation 2.22 .

${ }^{18}$ For this strategy to work it is important to write the first order conditions for the heterogeneous agents in equations (2.26)-(2.27) using the derivatives of the value functions and not as second order difference equations. For representative agents with no state contingent state variables it is often more convenient to write their first order conditions as second order difference equations. It is only for this reason that $x_{t}^{2}, x_{t+1}^{2}$ and $z_{t+1}$ are included in equation (2.27).

${ }^{19}$ In fact, I use the same notation as Uhlig (1999), page 38, to facilitate comparisons. The only difference is that the variables here written as $x_{t}^{1}$ and $y_{t+1}^{1}$ are there written as $x_{t-1}^{1}$ and $y_{t}^{1}$. However, in a deterministic context this difference is immaterial (it can be considered a simple notational issue).
} 
$\Phi_{21}=R_{21}, \Phi_{22}=R_{22}, \Gamma_{2}=S_{2}$, and

$$
\begin{aligned}
\Theta_{1} & =\Upsilon A_{21}^{-1} C_{21} K_{41}^{-1} J_{42} S_{2}, \\
\Psi_{1} & =\Upsilon\left[A_{21}^{-1} C_{21} K_{41}^{-1} J_{42} R_{22} Q_{2}+A_{21}^{-1} C_{21} K_{41}^{-1} K_{42} S_{2}+A_{21}^{-1} C_{21} K_{41}^{-1} M_{4}\right] \\
\Lambda_{1} & =-K_{41}^{-1} J_{42} R_{21} \Theta_{1}-K_{41}^{-1} J_{42} S_{2}, \\
\Gamma_{1} & =-K_{41}^{-1} J_{42} R_{21} \Psi_{1}-K_{41}^{-1} J_{42} R_{22} Q_{2}-K_{41}^{-1} K_{42} S_{2}-K_{41}^{-1} M_{4},
\end{aligned}
$$

where

$$
\Upsilon=\left[I-A_{21}^{-1} C_{21} K_{41}^{-1} J_{42} R_{21}\right]^{-1}
$$

Then, (2.29)-(2.32) solves the stochastic system (2.23)-(2.28).

Proof. The solution is verified using algebraic manipulations and the law of iterated expectations. ${ }^{20}$

\section{General applicability}

The computational method just described is applicable to a wide variety of models, but these models must satisfy certain conditions. A key feature of the computational method is that it uses a finite history of past decision rules to describe the current cross-sectional distributions of agents across individual states. For this strategy to work, the model considered should incorporate a significant life-cycle structure for the non-representative types of agents. In particular, their expected (or deterministic) lifetimes should be sufficiently short relative to the model time period. Otherwise, one may have to use prohibitively long histories of past decision rules in order to characterize the cross-sectional amount of heterogeneity accurately.

Another feature of the computational method is that it uses spline approximations to describe decision rules. This approach can accommodate a large class of decision rules but could become quite costly in certain cases. If the decision rules have ranges with sharp non-linearities, describing them accurately may require adding many grid points at those ranges. This could increase the computational costs significantly, since introducing more spline coefficients increases the number of aggregate state variables in the system (the computational method must keep track of the history of the additional coefficients). Another reason for the added complexity is that calculating

\footnotetext{
${ }^{20}$ See Technical Appendix 6 for a complete proof.
} 
numerical derivatives accurately at narrowly separated grid points requires having a good definition of the invariant distribution over the subranges that they define. ${ }^{21}$ Since the invariant distribution is obtained by performing Monte Carlo simulations, this may require working with a huge panel of agents. For these reasons, it is important to inspect the invariant distribution and decision rules at the deterministic steady state of the model and evaluate if the invariant distribution puts enough mass on ranges of non-linearities to justify the added complexity.

The spline decision rules will also only approximately describe the critical values at which a constraint becomes binding. Moreover, the computational method assumes that if a constraint binds (does not bind) at a given grid point in the deterministic steady state, that it will always bind (not bind) in the stochastic solution. ${ }^{22}$ While this assumption is likely to hold at most grid points, it may not hold at grid points that are sufficiently close to true critical values. In many cases these approximation errors will have unimportant consequences for the aggregate dynamics of the model. For example, if the invariant distribution puts little mass around the computed critical values, it will be largely irrelevant what happens in those ranges. Even if the invariant distribution puts significant mass around those critical values, the consequences of missing the associated constraints by small amounts are likely to be unimportant if the decision rules are sufficiently smooth. Problems may arise when the invariant distribution puts considerable mass close to the critical values and the decision rules are sharply non-linear around them. In these cases, the computational method may fail to capture the aggregate dynamics of the model correctly. Certain $(\mathrm{S}, \mathrm{s})$ economies fall in this category.

This does not mean that every $(\mathrm{S}, \mathrm{s})$ model is outside the scope of the computational method described in this paper. Many $(\mathrm{S}, \mathrm{s})$ models incorporate natural drifts that move agents away from the $(\mathrm{S}, \mathrm{s})$ thresholds, making the invariant distribution put zero mass around them. For example, these drifts could be generated by an exogenous depreciation rate of capital in an investment irreversibilities model (e.g. Veracierto (2002)), an exogenous quit rate of workers in a firm dynamics

\footnotetext{
${ }^{21}$ Recall the linearization of equation (2.13).

${ }^{22}$ In particular, for each grid point $\left(a, \bar{x}_{1 j}, \bar{x}_{2 j}\right)$ in the maximization problem described in (2.1), if some component of equation (2.4) holds with equality (strict inequality) at the deterministic steady state, the linearization performed in the construction of equation (2.12) implicitly imposes that this same component always holds with equality (strict inequality) at the stochastic solution.
} 
model with either firing costs or matching frictions (e.g. Veracierto (2008a), Veracierto (2016)), or an endogenous arrival rate of agents in an islands model with undirected search (e.g. Veracierto $(2008 b))$. In such models, the computational method described here could be applied perfectly well. In fact, this method is a generalization of the one used in the papers just mentioned. Instead of using spline approximations, the $(\mathrm{S}, \mathrm{s})$ adjustments in those papers allowed me to completely describe the decision rules with a finite number of $(\mathrm{S}, \mathrm{s})$ thresholds. Also, the drifts already mentioned made agents transit the $(\mathrm{S}, \mathrm{s})$ ranges of inaction in finite time and produced invariant distributions with finite support. As a consequence of this, I was able to describe the aggregate state of the economy using a finite history of $(\mathrm{S}, \mathrm{s})$ thresholds (or, equivalently, the finite support of the cross-sectional distribution). The computational method introduced in this paper generalizes that same approach to economies with general decision rules and cross-sectional distributions of agents with infinite support.

The rest of the paper illustrates the computational method using a model that has this more general structure. In particular, it illustrates the method using a Mirrlees RBC economy. ${ }^{23}$ There are two main reasons for this choice of model. The first reason is that the computational method can easily handle state-contingent distributions whereas other methods in the literature cannot. This difference calls for illustrating the computational method using a model with private information. The second reason is that in Veracierto (2019) I provide a sharp analytical characterization of the solution to the Mirrlees RBC economy considered here. Comparing the properties of the numerical solution to the theoretical results found in that paper provides an ideal test case for evaluating the accuracy of the method. It turns out that, while the computational method does not exploit the structure of the Mirrlees economy in any way, it recovers those analytical results exactly.

\footnotetext{
${ }^{23} \mathrm{~A}$ natural alternative would have been to use the Krusell et al. (1998) model, since it has been widely used in the literature. While the invariant distribution of that model generally puts positive mass at zero assets, the critical asset values at which agents start accumulating zero next-period assets have no mass in them. Moreover, the decision rules are quite smooth around those critical values (and approximately linear away from them.) Thus, the computational method could be perfectly applied (once the model is modified to incorporate some type of life cycle structure).
} 


\section{A Mirrlees RBC economy with known solution}

The economy is populated by a unit measure of agents subject to stochastic lifetimes. Whenever an agent dies they are immediately replaced by a newborn, leaving the aggregate population level constant over time. ${ }^{24}$ The preferences of an individual born at date $T$ are given by

$$
E_{T}\left\{\sum_{t=T}^{\infty} \beta^{t-T} \sigma^{t-T}\left[\ln \left(c_{t}\right)+\alpha_{t} \ln \left(1-h_{t}\right)\right]\right\},
$$

where $\sigma$ is the survival probability, $0<\beta<1$ is the discount factor, and $\alpha_{t} \in\left\{\alpha_{L}, \alpha_{H}\right\}$ is the idiosyncratic value of leisure (where $\alpha_{L}<\alpha_{H}$ ). Realizations of $\alpha_{t}$ are assumed to be i.i.d. both across individuals and across time. The probability that $\alpha_{t}=\alpha_{s}$ is given by $\psi_{s}$. A key assumption is that $\alpha_{t}$ is private information of the individual.

Output, which can be consumed or invested, is produced with the following production function:

$$
Y_{t}=e^{z_{t}} K_{t-1}^{\gamma} H_{t}^{1-\gamma}
$$

where $0<\gamma<1, Y_{t}$ is output, $z_{t}$ is aggregate productivity, $K_{t-1}$ is capital, and $H_{t}$ is hours worked. The aggregate productivity level $z_{t}$ follows a standard $\mathrm{AR}(1)$ process given by:

$$
z_{t+1}=\rho z_{t}+\varepsilon_{t+1}
$$

where $0<\rho<1$, and $\varepsilon_{t+1}$ is normally distributed with mean zero and standard deviation $\sigma_{\varepsilon}$.

Capital is accumulated using a standard linear technology given by

$$
K_{t}=(1-\delta) K_{t-1}+I_{t}
$$

where $I_{t}$ is gross investment and $0<\delta<1$.

\subsection{The mechanism design problem}

In what follows, I will describe the mechanism design problem for this economy. To do this, it will be convenient to distinguish between two types of agents: young and old. A young agent is one that has been born at the beginning of the current period. An old agent is one that has been

\footnotetext{
${ }^{24}$ As in Phelan (1994), the stochastic lifetime guarantees that there will be a stationary distribution of agents across individual states.
} 
born in some previous period. The social planner must decide recursive plans for both types of agents. The state of a recursive plan is the value (i.e., discounted expected utility) that the agent is entitled to at the beginning of the period. Given this promised value, the recursive plan specifies the current utility of consumption, the current utility of leisure, and next-period promised values as functions of the value of leisure currently reported by the agent. The social planner is fully committed to the recursive plans they choose and agents have no outside opportunities available.

A key difference between the young and the old is in terms of promised values. Since during the previous period the social planner has already decided on some recursive plan for a currently old agent, the planner is restricted to delivering the corresponding promised value during the current period. In contrast, the social planner is free to deliver any value to a currently young agent since this is the first period they are alive. Reflecting this difference, I will specify the individual state of an old agent to be their promised value $v$ and their current value of leisure $s$ (henceforth, I will refer to the value of leisure $\alpha_{s}$ by its subindex $\left.s \in\{L, H\}\right)$. At date $t$, their current utility of consumption, utility of leisure, and next-period promised value are denoted by $u_{o s t}(v), n_{o s t}(v)$ and $w_{o s, t+1}(v)$, respectively, where $w_{o s, t+1}(v)$ is a random variable contingent on the realization of $z_{t+1}$. In turn, the individual state of a young agent is solely given by their current value of leisure $s$. At date $t$, the agent's current utility of consumption, utility of leisure, and next-period promised value are denoted by $u_{y s t}, n_{y s t}$ and $w_{y s, t+1}$ respectively, where $w_{y s, t+1}$ is also contingent on the realization of $z_{t+1}$.

The social planner seeks to maximize the weighted sum of the welfare levels of the current and future generations of young agents, subject to individual incentive compatibility and promise keeping constraints, as well as aggregate feasibility constraints. ${ }^{25}$ Veracierto (2019) describes this economy-wide planning problem in detail. However, in order to map the mechanism design problem into the structure described in Section 2, it will be convenient to decompose that planning problem into a sequence of sub-planning problems. In each period, there are two sub-planning problems: one sub-planning problem concerned with providing insurance and incentives to individuals, and another sub-planning problem concerned with making production and investment decisions. In these sub-planning problems, the joint stochastic process for the shadow price of

\footnotetext{
${ }^{25}$ The welfare levels of the current old agents are predetermined by their promised values at the beginning of the period.
} 
labor (in terms of the consumption good), $q_{t}$, and the shadow price of the consumption good (in utiles), $\lambda_{t}$, are taken as given. The solutions to these sequences of sub-planning problems correspond to those of the economy-wide planning problem if certain side conditions are satisfied.

The sub-planning problems for individuals differ depending on whether the individual is young or old. For every date $t$, the sub-planning problem for old individuals is as follows:

$$
P_{o t}(v)=\max \sum_{s} \psi_{s}\left\{q_{t} h\left(n_{o s t}\right)-c\left(u_{o s t}\right)+\theta \sigma E_{t}\left[\frac{\lambda_{t+1}}{\lambda_{t}} P_{o, t+1}\left(w_{o s, t+1}\right)\right]\right\}
$$

subject to

$$
\begin{gathered}
u_{o L t}+\alpha_{L} n_{o L t}+\beta \sigma E_{t}\left[w_{o L, t+1}\right] \geq u_{o H t}+\alpha_{L} n_{o H t}+\beta \sigma E_{t}\left[w_{o H, t+1}\right], \\
v=\sum_{s}\left\{u_{o s t}+\alpha_{s} n_{o s t}+\beta \sigma E_{t}\left[w_{o s, t+1}\right]\right\} \psi_{s}
\end{gathered}
$$

where $h(n)$ are the hours worked implied by the utility of leisure $n$ (i.e. $h(n)=1-e^{n}$ ), and $c(u)$ is the consumption level implied by the utility of consumption $u$ (i.e. $c(u)=e^{u}$ ). Observe that the current "social profits" in equation (4.5) are given by the social value of the hours worked by the old agent, net of the consumption goods that are transferred to them. Also observe that the sub-planner discounts the future social profits of the old individual using the social discount factor $\theta$, the survival probability $\sigma$, and the stochastic social discount factor $\lambda_{t+1} / \lambda_{t}$. The social discount rate $\theta$ is the Pareto weight of the next-period generation of young agents relative to the Pareto weight of the current generation of young agents. ${ }^{26}$ Equation (4.6) is the binding incentive compatibility constraint. It states that the expected value to the individual of truthfully reporting the low value of leisure $L$ must be at least as large as the expected value to the individual of misreporting the high value of leisure $H$. Equation (4.7) is the promise-keeping constraint. It states that the social sub-planner must deliver the expected value $v$ that was promised at the beginning of the period.

For every date $t$, the sub-planning problem for young individuals is as follows:

$P_{y t}=\max \sum_{s} \psi_{s}\left\{\frac{u_{y s t}+\alpha_{s} n_{y s t}+\beta \sigma E_{t}\left[w_{y s, t+1}\right]}{\lambda_{t}}+q_{t} h\left(n_{y s t}\right)-c\left(u_{y s t}\right)+\theta \sigma E_{t}\left[\frac{\lambda_{t+1}}{\lambda_{t}} P_{o, t+1}\left(w_{y s, t+1}\right)\right]\right\}$

subject to

$$
u_{y L t}+\alpha_{L} n_{y L t}+\beta \sigma E_{t}\left[w_{y L, t+1}\right] \geq u_{y H t}+\alpha_{L} n_{y H t}+\beta \sigma E_{t}\left[w_{y H, t+1}\right]
$$

\footnotetext{
${ }^{26} \mathrm{I}$ assume that $\beta \sigma<\theta<1$.
} 
Observe that in this case the social surplus is given by the expected lifetime utility level of the young agent (in current consumption units), plus the expected social value of the hours worked by the agent, net of the expected consumption goods transferred to them. Since, conditional on surviving the young agent becomes old after one period, the function used to evaluate next-period continuation values is $P_{o, t+1}$.

For every date $t$, the sub-planning problem for production decisions is

$$
P_{p t}(K)=\max \left\{e^{z_{t}} K^{\gamma} H_{t}^{1-\gamma}-q_{t} H_{t}-I_{t}+\theta E_{t}\left[\frac{\lambda_{t+1}}{\lambda_{t}} P_{p, t+1}\left((1-\delta) K+I_{t}\right)\right]\right\} .
$$

Observe that the social surplus generated by this planning problem is given by output net of the value of the labor input and the value of investment.

The economy-wide distribution of old agents across promised values $v$ at the beginning of period $t$ is given by a measure $\mu_{t}$, while the number of young agents is constant over time and given by $1-\sigma$. Given the stochastic sequence of decision rules $\left[u_{o s t}, n_{o s t}, w_{o s, t+1}, u_{y s t}, n_{y s t}, w_{y s, t+1}\right]_{s}$ that solve the corresponding sub-planning problems for individuals, the law of motion for $\mu_{t}$ is given as follows:

$$
\mu_{t+1}(B)=\sigma \sum_{s} \int_{\left\{v: w_{o s, t+1}(v) \in B\right\}} \psi_{s} d \mu_{t}+(1-\sigma) \sigma \sum_{s: w_{y s, t+1} \in B} \psi_{s}
$$

for every Borel set B. Equation (4.11) states that the number of old agents that have a promised value in the Borel set $B$ at the beginning of the following period is given by the sum of two terms. The first term sums all currently old agents that receive a next-period promised value in the set $B$ and do not die. The second term does the same for all currently young agents.

The economy-wide stock of capital at the beginning of period $t$ is equal to $K_{t-1}$. Given the stochastic sequence of decision rules $\left[H_{t}, I_{t}\right]$ that solve the sub-planning production problems, $K_{t}$ follows a stochastic process given by

$$
K_{t}=(1-\delta) K_{t-1}+I_{t}\left(K_{t-1}\right)
$$

The side conditions that the stochastic shadow prices $\left\{q_{t}, \lambda_{t}\right\}_{t=1}^{\infty}$ need to satisfy at every date $t$ are the following:

$$
(1-\sigma) \sum_{s} c\left(u_{y s t}\right) \psi_{s}+\int \sum_{s} c\left(u_{o s t}(v)\right) \psi_{s} d \mu_{t}+I_{t}\left(K_{t-1}\right)=e^{z_{t}} K_{t-1}^{\gamma} H_{t}\left(K_{t-1}\right)^{1-\gamma}
$$


and

$$
H_{t}\left(K_{t-1}\right)=(1-\sigma) \sum_{s} h\left(n_{y s t}\right) \psi_{s}+\int \sum_{s} h\left(n_{\text {ost }}(v)\right) \psi_{s} d \mu_{t} .
$$

Equation (4.13) describes the aggregate feasibility constraint for the consumption good. It states that the total consumption of young and old agents, plus aggregate investment cannot exceed aggregate output. Equation (4.14) is the aggregate labor feasibility constraint. It states that the input of hours into the production function cannot exceed the total hours worked by young and old agents.

\subsection{Applying the general computational method}

In this section I show that the mechanism design problem described above has the general structure described in Section 2. As a consequence, the deterministic steady state optimal allocation can be computed as in Section 2.1 and the stationary stochastic optimal allocation can be computed as in Section 2.2

There are two permanent types $h$ of agents in this economy: production sub-planners and individuals sub-planners. The production sub-planner has a "representative" type so I denote it with subscript $r$. The $h$ subscript is reserved for individuals sub-planners.

There are no $z_{t}$-contingent states $x_{1 r t}$ for production sub-planners (observe that the notation for aggregate shocks $z_{t}$ coincides with the general setting). However, it does have a non-contingent state: the stock of capital. Thus, $x_{2 r, t-1} \equiv K_{t-1}$. The vector of decision functions $u_{2 r t}$ is given by $\left(H_{t}, I_{t}\right)$. The function $G_{r 2}$ in equation (2.8) is then given by equation (4.12). The counterpart for the maximization problem given by equations (2.1)-(2.4) is equation (4.10), with $v_{r t} \equiv P_{p t}$, $x_{2} \equiv K$, there is no $a, x_{1}$ or $s, p_{t}=\left(q_{t}, \lambda_{t}\right)$, and

$$
\begin{aligned}
R_{r} & \equiv e^{z_{t}} K^{\gamma} H_{t}^{1-\gamma}-q_{t} H_{t}-I_{t} \\
\beta_{r} & \equiv \theta \frac{\lambda_{t+1}}{\lambda_{t}} \\
G_{r 2} & \equiv(1-\delta) K+I_{t} .
\end{aligned}
$$

Observe that there is no equation (2.2) or (2.4).

For individuals sub-planners there are two values for $a: y$ (young) and o (old). There is no uncontingent state $x_{2}$, and the $z_{t}$-contingent state is the promised value, i.e. $x_{1} \equiv v$. Observe that $\pi_{h}(y, y)=0, \pi_{h}(y, o)=\sigma, \pi_{h}(o, o)=\sigma$ and $\pi_{h}(o, y)=0$ (these transition probabilities are 
independent of individual decisions). The idiosyncratic shock $s$ corresponds to the index of the idiosyncratic value of leisure $\alpha_{s}$ (the notation for the probabilities $\psi_{s}$ coincides in both contexts).

When the sub-planner is deciding over an old individual (i.e. $a=o$ ), the maximization problem in equations (2.1)-(2.4) is described by equations (4.5)-(4.7). Thus, $v_{h t} \equiv P_{o t}$, the $z_{t+1}$-decision variables $\left[u_{h 1, t+1}\left(s, a^{\prime}\right)\right]_{a^{\prime}}$ are given by $w_{o s, t+1}$ and the uncontingent decision variables $\left[u_{h 2 t}\left(s, a^{\prime}\right)\right]_{a^{\prime}}$ are given by $\left(u_{o s t}, n_{o s t}\right)$. Also,

$$
\begin{aligned}
R_{h} & \equiv q_{t} h\left(n_{o s t}\right)-c\left(u_{o s t}\right) \\
\beta_{h} & \equiv \theta \frac{\lambda_{t+1}}{\lambda_{t}} \\
G_{h 1} & =w_{o s, t+1}
\end{aligned}
$$

and $C_{h}$ in equation (2.4) is given by equations (4.6)-(4.7). There is no $G_{h 2}$.

When the sub-planner is deciding over a young individual (i.e. $a=y$ ), the maximization problem in equations (2.1)-(2.4) is described by equations (4.8)-(4.9). Thus, $v_{h t} \equiv P_{y t}$, the $z_{t+1^{-}}$ decision variables $\left[u_{h 1, t+1}\left(s, a^{\prime}\right)\right]_{a^{\prime}}$ are given by $w_{y s, t+1}$ and the uncontingent decision variables $\left[u_{h 2 t}\left(s, a^{\prime}\right)\right]_{a^{\prime}}$ are given by $\left(u_{y s t}, n_{y s t}\right)$. Also,

$$
\begin{aligned}
R_{h} & \equiv \frac{u_{y s t}+\alpha_{s} n_{y s t}+\beta \sigma w_{y s, t+1}}{\lambda_{t}}+q_{t} h\left(n_{y s t}\right)-c\left(u_{y s t}\right) \\
\beta_{h} & \equiv \theta \frac{\lambda_{t+1}}{\lambda_{t}} \\
G_{h 1}(a, s) & =w_{y s, t+1}
\end{aligned}
$$

and $C_{h}$ in equation (2.4) is given by equation (4.9). There is no $G_{h 2}$.

The distribution $\mu_{h t}$ of $h$-type agents across individual states $\left(a, x_{1}, x_{2}\right)$ at the beginning of period $t$ is described by the measure $\mu_{t}$ when $a=o$ and by the total number of young agents $1-\sigma$ when $a=y$ (since the decision rules of young agents do not depend on promised values, there is no need to specify an explicit measure across promised values for young agents). The law of motion in equation (2.5) for $a^{\prime}=o$ is then given by equation (4.11), with $\phi_{h}\left(\left\{a^{\prime}\right\} \times \mathcal{X}_{1}\right)=0$.

Finally, the vector valued function $Q$ in equation (2.9) is given by equations (4.13) and (4.14). The moments in $M_{h}$ that enter the first component of $Q$ are $c\left(u_{y s t}\right)$ and $c\left(u_{o s t}(v)\right)$, and the moments that enter the second component of $Q$ are $h\left(n_{y s t}\right)$ and $h\left(n_{\text {ost }}(v)\right) .{ }^{27}$

\footnotetext{
${ }^{27}$ While this section has shown how to map the Mirrlees RBC economy to the general structure of Section 2 ,
} 


\subsection{Testing the computational method}

Having shown how to map the Mirrlees RBC economy into the general structure of Section 2, I use it to test the accuracy of the computational method. The reason, as has already been mentioned, is that in Veracierto (2019) I establish key features of the stationary solution analytically. In particular, I demonstrate that under the logarithmic preferences assumed here that the following properties hold:

Property 1: $u_{y s t}, n_{y s t}$, and $w_{y s, t+1}$ fluctuate over the business cycle by amounts that are independent of the reported type $s$,

Property 2: each of the allocation rules $u_{o s t}(v), n_{o s t}(v)$, and $w_{o s, t+1}(v)$ are strictly increasing linear functions that are parallel across reported types,

Property 3: $u_{o s t}(v), n_{o s t}(v)$, and $w_{o s, t+1}(v)$ shift over the business cycle while keeping their slopes constant, and the shifts are independent of the reported type $s$,

Property 4: the cross-sectional distributions of promised values $v$, of log-consumption $u_{\text {ost }}$, and of $\log$-leisure $n_{\text {ost }}$, shift horizontally over the business cycle while maintaining their shapes,

Property 5: aggregate consumption $C_{t}$, aggregate hours worked $H_{t}$, and aggregate capital $K_{t}$ are exactly the same as in the stationary solution to the following representative agent planning problem:

$$
V\left(z_{t}, K_{t-1}\right)=\max \left\{u\left(C_{t}\right)+\bar{\alpha} n\left(1-H_{t}\right)+\theta E_{t}\left[V\left(z_{t+1}, K_{t}\right)\right]\right\}
$$

subject to

$$
C_{t}+K_{t}-(1-\delta) K_{t-1} \leq e^{z_{t}} K_{t-1}^{\gamma} H_{t}^{1-\gamma}
$$

where $\bar{\alpha}=\alpha_{L} \psi_{L}+\alpha_{H} \psi_{H}$. I refer the reader to Veracierto (2019) for the economics behind these results, as well as for results corresponding to other preferences. What I am interested here is to show that the computational method recovers the above properties exactly. In order to do this I must first parametrize the model.

Following the RBC literature, I select a labor share $1-\gamma$ of 0.64 , a depreciation rate $\delta$ of 0.10 , a private discount factor $\beta$ of 0.96 , a persistence of aggregate productivity $\rho$ of 0.95 , and a variance

the reader interested in implementing this method to other models may want to review Technical Appendix 7. This appendix is rich in concrete implementation details, such as listing each of the first order conditions and their arguments, and classifying each first order condition into one of the five types given by equations (2.23)-(2.27). The appendix also shows how the general linearized model (2.23)-(2.28) simplifies in this particular application. 
of the innovations to aggregate productivity $\sigma_{\varepsilon}^{2}$ equal to $4 \times 0.007^{2}$, all corresponding to a time period of one year. The social discount factor $\theta$ is chosen to be the same as the private discount factor $\beta$. The values of leisure $\alpha_{L}$ and $\alpha_{H}$ are chosen to satisfy two criteria: That aggregate hours worked $H$ equal 0.31 (a standard target in the RBC literature) and that the hours worked by old agents with the high value of leisure and the highest possible promised value $n_{o H}\left(v_{\max }\right)$ be a small but positive number. The rationale for this second criterion is that I want to maximize the relevance of the information frictions while keeping an internal solution for hours worked. The resulting values for $\alpha_{L}$ and $\alpha_{H}$ are 1.643 and 2.177, respectively. I treat both values of the idiosyncratic shock symmetrically and chose $\psi_{L}=\psi_{H}=0.50$. In terms of the life-cycle structure, I choose $\sigma=0.975$ to generate an expected lifespan of 40 years.

While the above parameters are structural, there are a number of computational parameters to be determined. The number of grid points in the spline approximations $J$, the total number of agents simulated $I$, the length of the simulations for computing the invariant distribution $T$, and the length of the histories kept as state variables when computing the business cycles $N$ are all chosen to be as large as possible, while keeping the computational task manageable and results being robust to non-trivial changes in their values. Their chosen values are 20, $2^{23}, 1000$, and 273 , respectively. ${ }^{28}$ It turns out that under these computational parameters, the linearized system described in Section 2.3 has about 12,000 variables (a large system indeed).

Finally, the lower and upper bounds for the range of possible promised values $v_{\min }$ and $v_{\max }$ were chosen so that the fraction of agents in the intervals $\left[v_{1}, v_{2}\right]$ and $\left[v_{J-1}, v_{J}\right]$ are each less than $0.01 \%$. Thus, truncating the range of possible values at $v_{\min }$ and $v_{\max }$ should not play an important role in the results. The chosen values for $v_{\min }$ and $v_{\max }$ are -35.0 and -16.3 , respectively.

Before turning to the business cycle results, I illustrate different features of the model at its deterministic steady state. Figure 1 shows the invariant distribution of promised values across the $J-1$ intervals $\left[v_{j}, v_{j+1}\right]_{j=1}^{J-1}$, defined by the grid points of the spline approximations. We see that the invariant distribution puts very little mass at extreme values. In consequence, in what follows I will report allocation rules only between the 6 th and 16 th grid points. The reason is not only

\footnotetext{
${ }^{28}$ Given the value selected for the survival probability $\sigma$, less than $0.1 \%$ of individuals survive more than $N$ periods. Thus, the truncation imposed by keeping track of a finite history of decision rules introduces a very small approximation error.
} 
that there are too few agents at the tails of the distribution for them to matter, but also that being close to the artificial bounds $v_{\min }$ and $v_{\max }$ greatly distorts the shape of the allocation rules.

Figure 2.A reports utilities of consumption for old agents $u_{o L}(v)$ and $u_{o H}(v)$ across promised values $v$, at the deterministic steady state. We see that both $u_{o L}$ and $u_{o H}$ are strictly increasing in the promised value $v$, are linear, and are parallel to each other. Figure 2.B shows the same for the utilities of leisure $n_{o L}(v)$ and $n_{o H}(v)$, and Figure 2.C for the next-period promised values $w_{o L}(v)$ and $w_{o H}(v)$. Since these figures could be tricking the naked eye, Figure 2.D, depicts the vertical differences across reported types $u_{o H}(v)-u_{o L}(v), n_{o H}(v)-n_{o L}(v)$ and $w_{o H}(v)-w_{o L}(v)$. We see that the different pairs of functions are indeed parallel to each other. Thus, Figure 2 verifies that Property 2 holds at the deterministic steady state.

The discussion of business cycle dynamics that follows is centered around the analysis of the impulse responses of different variables to a one standard deviation increase in aggregate productivity. Figure 3.A shows the impulse responses of the utility of consumption of young agents $u_{y L}$ and $u_{y H}$. We see that the two impulse responses overlap perfectly, thus satisfying Property 1. Figure 3.B shows the impulse response of the utility of consumption of old agents with a low value of leisure $u_{o L}(v)$, at each of the eleven grid points $\left(v_{j}\right)_{j=6}^{16}$. While the figure shows eleven impulse responses, only one of them is actually seen because they overlap perfectly. This means that, in response to the aggregate productivity shock, the linear function $u_{o L}$ depicted in Figure 2.A shifts vertically over time while keeping its slope constant. Figure 3.C, which does the same for $u_{o H}$, is identical to Figure 3.B. Thus, not only $u_{o H}$ shifts over time keeping its slope constant, but its increments are the same as those of $u_{o L}$. We have thus verified that $u_{o L t}$ and $u_{o H t}$ satisfy Property 3. Figure 4 is analogous to Figure 3, except that it depicts the behavior of the utilities of leisure $n_{y s t}$ and $\left[n_{o s t}\left(v_{j}\right)\right]_{j=6}^{16}$. Figure 5 is also analogous to Figure 3 but depicts the behavior of the promised values $w_{y s, t+1}$ and $\left[w_{o s, t+1}\left(v_{j}\right)\right]_{j=6}^{16}$. A quick inspection verifies that Figures 4 and 5 have the same characteristics as Figure 3. Thus, Properties 1 and 3 are fully satisfied.

Figure 6 shows the impulse responses of the cross sectional standard deviations of promised values, log-consumption and log-leisure. We see that in response to a positive aggregate productivity shock, all these standard deviations remain flat. Thus, Property 4 is satisfied.

Finally, Figure 7.A shows the impulse responses of aggregate output $Y_{t}$, aggregate consumption $C_{t}$, aggregate investment $I_{t}$, aggregate hours worked $H_{t}$ and aggregate capital $K_{t-1}$ for the Mirrlees 
RBC economy. Figure 7.B reports the impulse responses for the same variables but for the representative agent economy planning problem (4.15)-(4.16). We see that Figures 7.A and 7.B are the same. Figure 7.C verifies this by reporting the differences between the Mirrlees economy and the representative agent economy, for each of the macro variables considered. Thus, Property 5 is perfectly satisfied.

We have thus verified that the general computational method, when applied to the Mirrlees RBC economy with logarithmic preferences, reproduces all the analytical properties found in Veracierto (2019). Since nothing in the computational method exploits the functional forms or structure of the economy considered, this provides significant evidence about its accuracy. This finding indicates that the computational method introduced in this paper should prove useful in a variety of other settings.

\section{Conclusions}

In this paper I introduced a general method for computing equilibria of economies with heterogeneous agents and aggregate shocks. Its basic strategy is to parametrize individual decision rules as spline approximations and to keep long histories of the spline coefficients as state variables. The resulting representation of the model is then linearized at the deterministic steady-state. Three important features make this approach attractive as a general computational method: 1) it keeps track of the full distribution of agents across individual states, 2) it can handle irregular shapes for this distribution, and 3) it incorporates the distribution's exact law of motion. In addition, the computational method is able to handle cases in which the cross-sectional distribution of agents is state-contingent. This last property makes the method particularly useful for computing aggregate fluctuations of economies with private information.

The computational method was then illustrated using a Mirrlees RBC economy with known analytical solution. Contrasting the numerical solution to the theoretical solution allowed me to test the accuracy of the computational method. The method passed the test with flying colors: it reproduced all the theoretical properties of the solution perfectly well. This finding suggests that the computational method should prove useful in a variety of other applications.

While the features of the computational method mentioned above are extremely attractive, I would like to conclude the paper with two caveats. The first one is that, since linearizing the 
model with respect to each of the elements in the history of spline coefficients requires performing a massive number of Monte Carlo simulations, the method turns out to be rather slow. ${ }^{29}$ This should not be a problem when calibrating the deterministic steady-state of a model, since the computational method needs to be applied only once (after all parameter values have been determined). However, it makes it impractical for estimating a model using formal econometric methods. The second caveat is that the computational complexity grows exponentially with the number of endogenous individual state variables. The reason is that as the spline approximations are defined over state spaces of increasing dimensionality, the number of spline coefficients in the system grow accordingly. As a result, models with two endogenous individual state variables could only be handled if the decision rules are sufficiently smooth to be described with a relatively small number of grid points. Models with two endogenous state variables and significant non-linearities, or with more than two endogenous individual state variables are currently outside the scope of the method.

\section{References}

Achdou, Y., J. Han, J.-M. Lasry, P.-L. Lions and B. Moll, "Income and Wealth Distribution in Macroeconomics: A Continuous-Time Approach," NBER Working Papers 23732, National Bureau of Economic Research, Inc, August 2017.

Ahn, S., G. Kaplan, B. Moll, T. Winberry and C. Wolf, "When Inequality Matters for Macro and Macro Matters for Inequality," NBER Macroeconomics Annual 32 (2018), 1-75.

Algan, Y., O. Allais And W. J. Den HaAn, "Solving heterogeneous-agent models with parameterized cross-sectional distributions," Journal of Economic Dynamics and Control 32 (March 2008), 875-908.

Algan, Y., O. Allais, W. J. D. HaAn and P. Rendhal, "Solving and Simulating Models with Heterogeneous Agents and Aggregate Uncertainty," in K. Schmedders and K. L. Judd, eds., Handbook of Computational Economicsvolume 3 (North-Holland, 2014), 277-324.

\footnotetext{
${ }^{29}$ Linearizing the Mirlees RBC model under the computational parameters described in Section 4.3 takes about six hours in a system equipped with two NVIDIA Tesla V100 GPUs.
} 
Boppart, T., P. Krusell and K. Mitman, "Exploiting MiT shocks in heterogeneous-agent economies: the impulse response as a numerical derivative," Journal of Economic Dynamics and Control 89 (2018), 68-92.

Campbell, J., "Entry, Exit, Embodied Technology, and Business Cycles," Review of Economic Dynamics 1 (April 1998), 371-408.

Den HaAn, W. J., "Heterogeneity, Aggregate Uncertainty, and the Short-Term Interest Rate," Journal of Business 83 Economic Statistics 14 (October 1996), 399-411.

—_, "Solving Dynamic Models With Aggregate Shocks And Heterogeneous Agents," Macroeconomic Dynamics 1 (June 1997), 355-386.

Dotsey, M., R. G. King And A. L. Wolman, "State-Dependent Pricing and the General Equilibrium Dynamics of Money and Output," The Quarterly Journal of Economics 114 (1999), 655-690.

Krusell, P., A. A. Smith And JR., "Income and Wealth Heterogeneity in the Macroeconomy," Journal of Political Economy 106 (October 1998), 867-896.

Mertens, T. M. And K. L. JudD, "Solving an incomplete markets model with a large crosssection of agents," Journal of Economic Dynamics and Control 91 (2018), 349-368.

Phelan, C., "Incentives and Aggregate Shocks," Review of Economic Studies 61 (1994), 681-700.

Preston, B. And M. Roca, "Incomplete Markets, Heterogeneity and Macroeconomic Dynamics," NBER Working Papers 13260, National Bureau of Economic Research, Inc, July 2007.

Reiter, M., "Solving heterogeneous-agent models by projection and perturbation," Journal of Economic Dynamics and Control 33 (March 2009), 649-665.

Uhlig, H., "A Toolkit for Analysing Nonlinear Dynamic Stochastic Models Easily," in R. Marimon and A. Scott, eds., Computational Methods for the Study of Dynamics Economies (Oxford: Oxford University Press, 1999), 30-61.

Veracierto, M. L., "Plant-Level Irreversible Investment and Equilibrium Business Cycles," American Economic Review 92 (2002), 181-197. 
— - "Firing Costs And Business Cycle Fluctuations," International Economic Review 49 (2008a), 1-39.

—_ "On the cyclical behavior of employment, unemployment and labor force participation," Journal of Monetary Economics 55 (2008b), 1143-1157.

—_, "Establishment Dynamics, Vacancies, And Unemployment: A Neoclassical Approach," International Economic Review 57 (November 2016), 1201-1236.

—, "Business Cycle Fluctuations in Mirrlees Economies: The case of i.i.d. shocks," mimeo, Federal Reserve Bank of Chicago, 2019.

WinBERRY, T., "A method for solving and estimating heterogeneous agent macro models," Quantitative Economics 9 (November 2018), 1123-1151. 
Figure 1: Invariant distribution of promised values

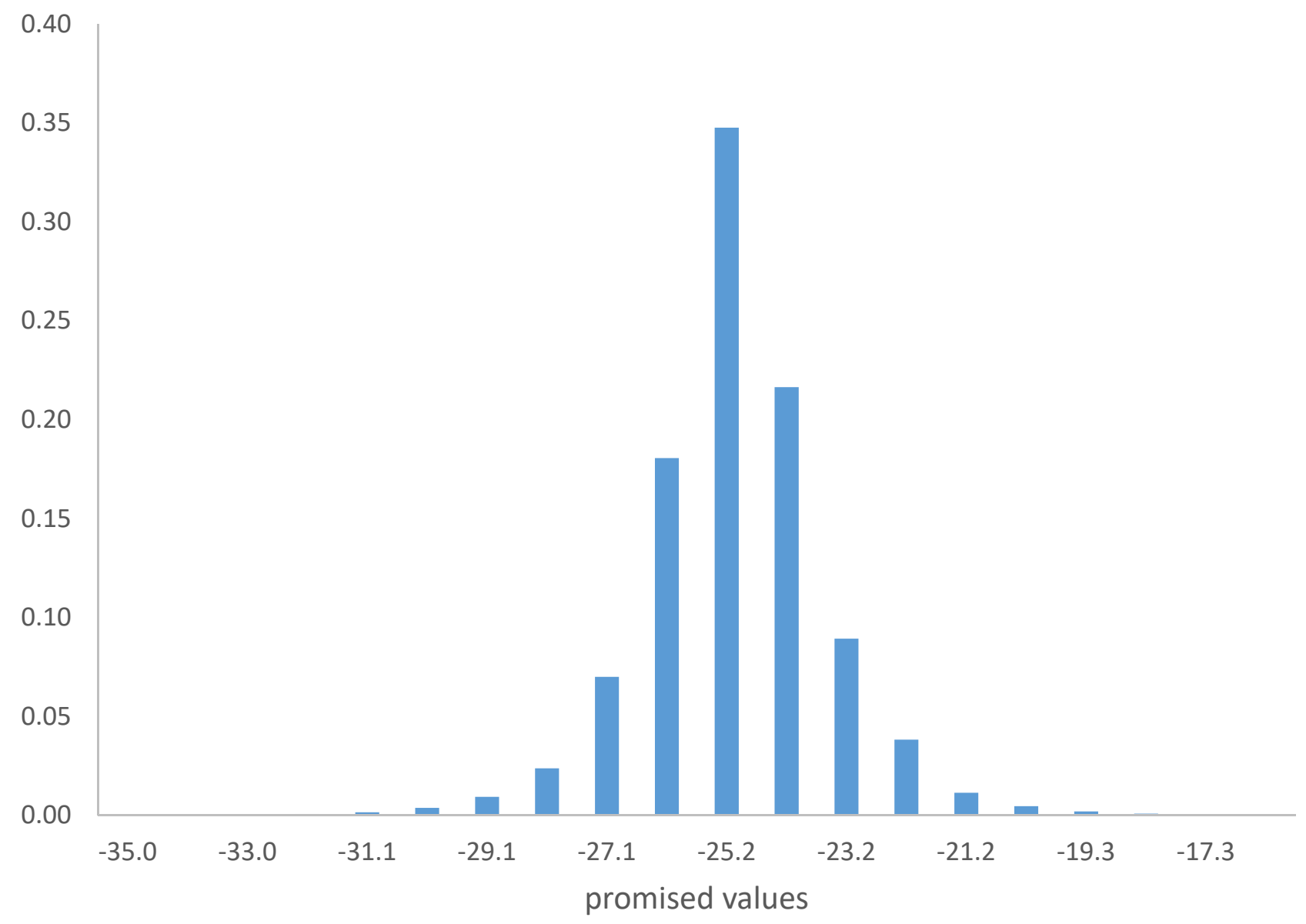


Figure 2: Steady state allocation rules
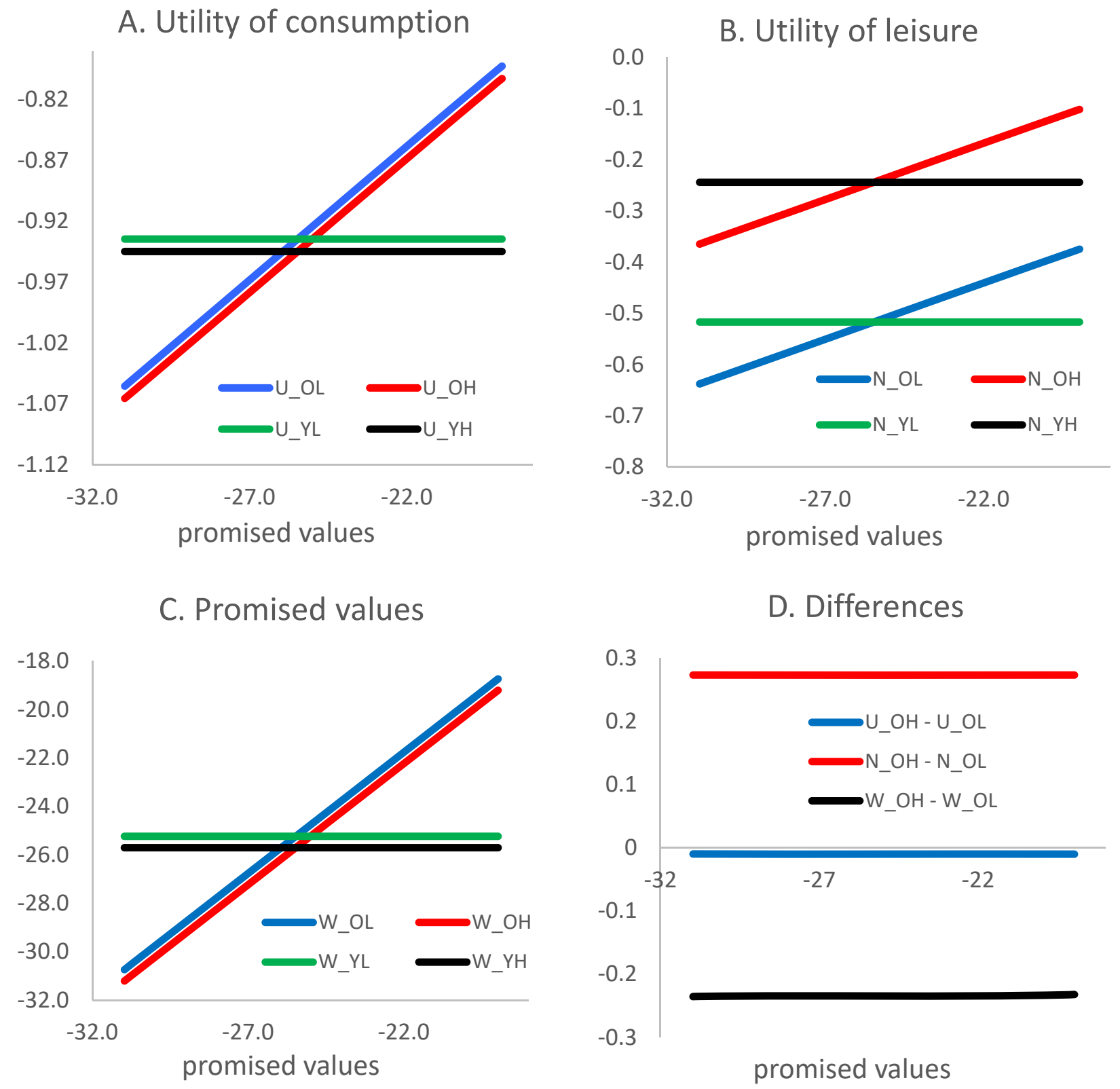
Figure 3: Impulse responses for consumption utilities

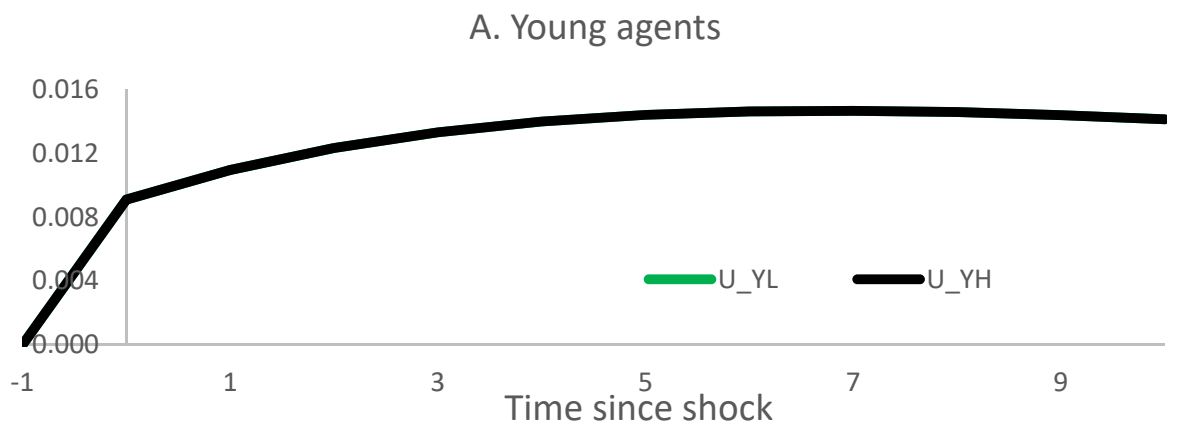

B. Old agents with low value of leisure

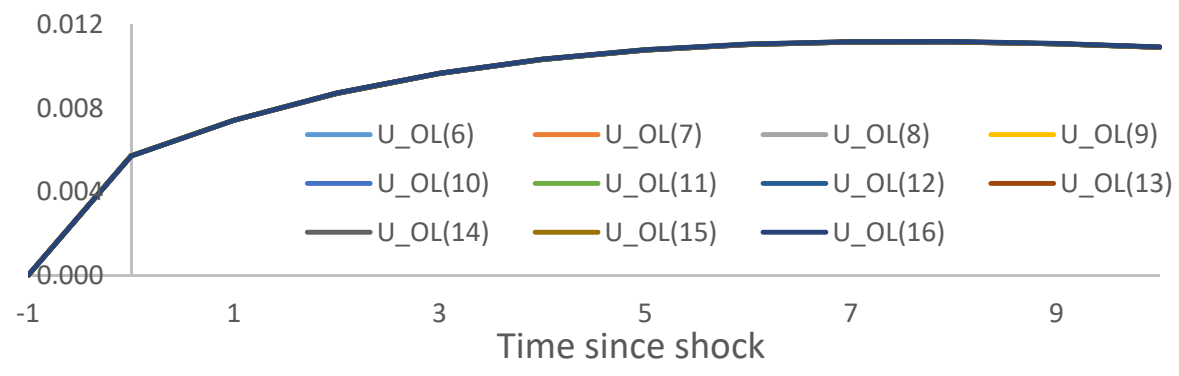

C: Old agents with high value of leisure

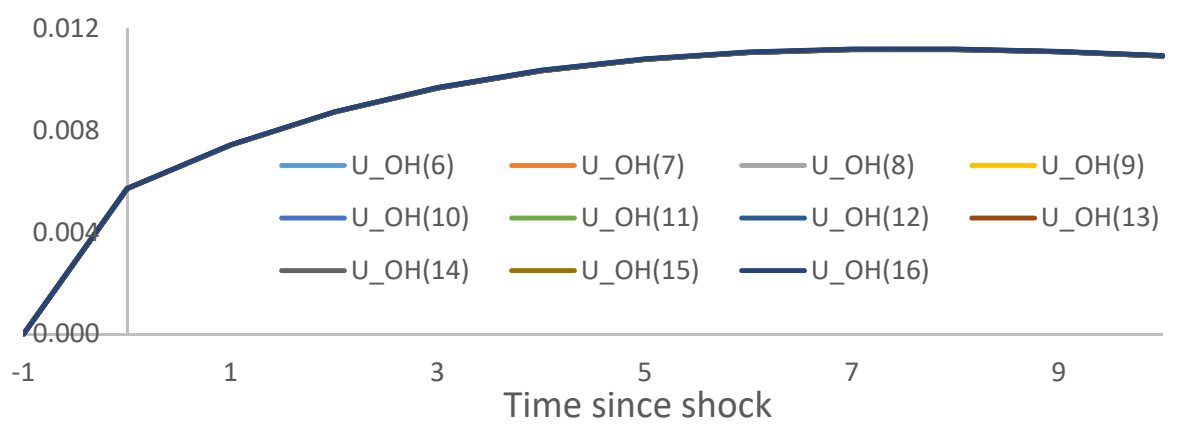


Figure 4: Impulse responses for leisure utilities

\section{A. Young agents}

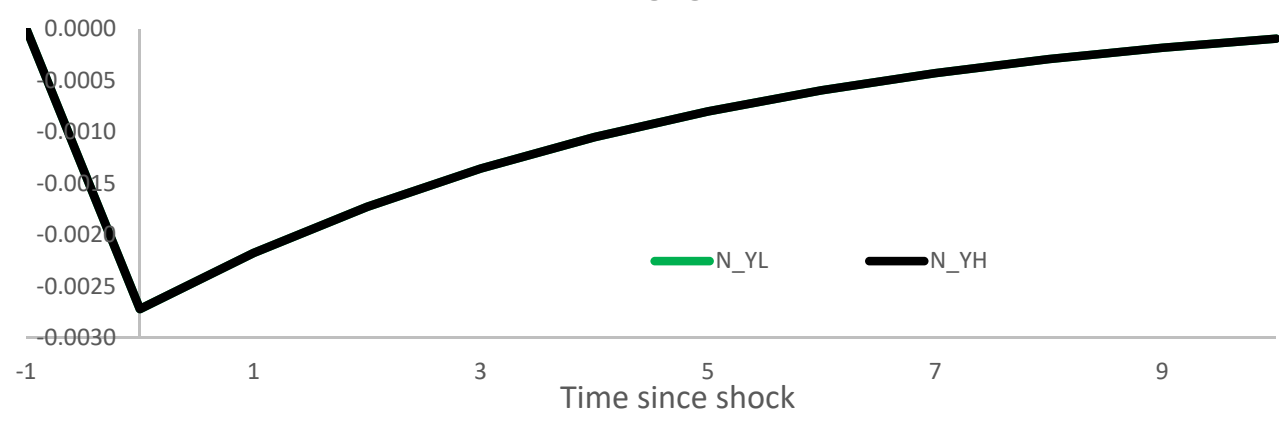

B. Old agents with low value of leisure

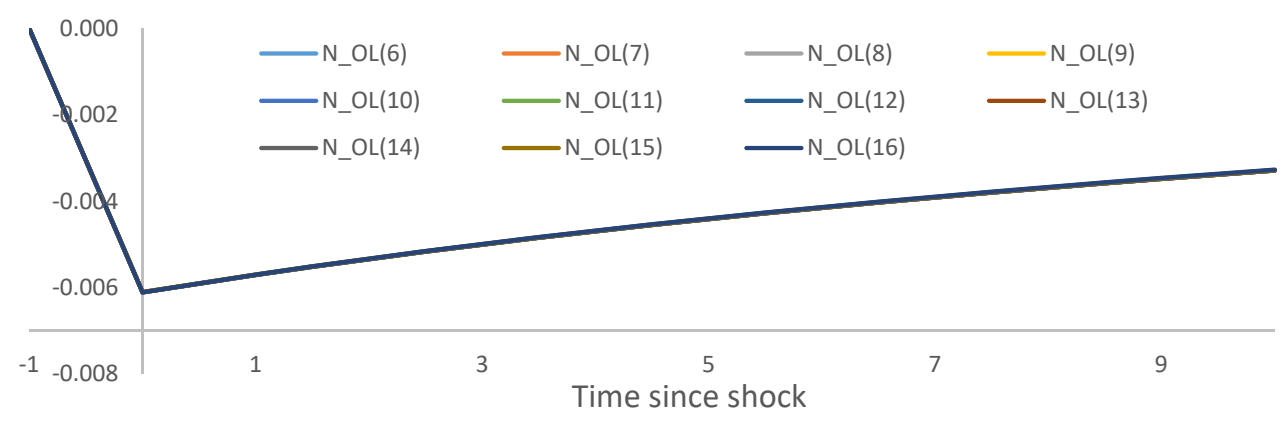

C. Old agents with high value of leisure

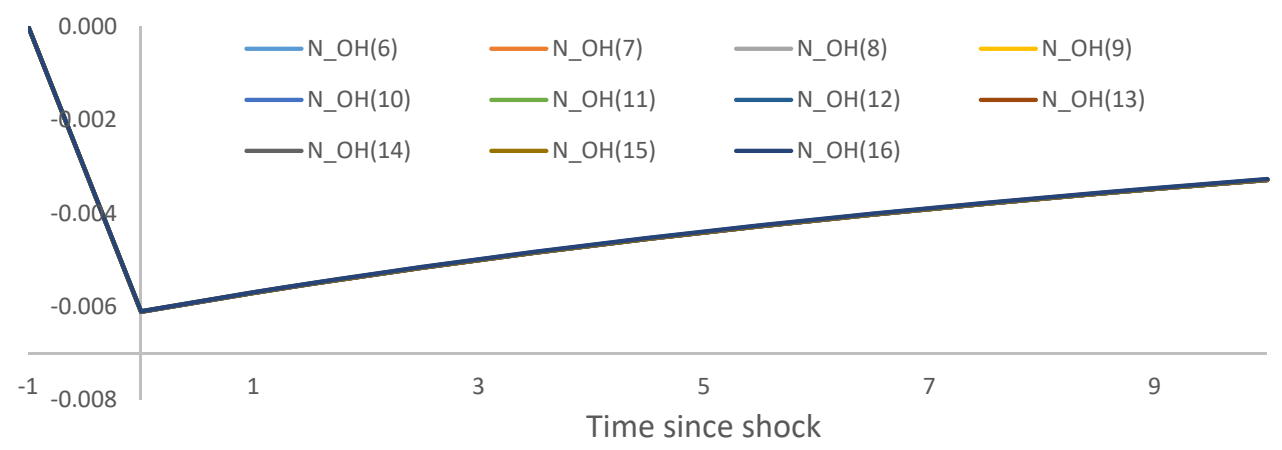


Figure 5: Impulse responses for promised values

A. Young agents

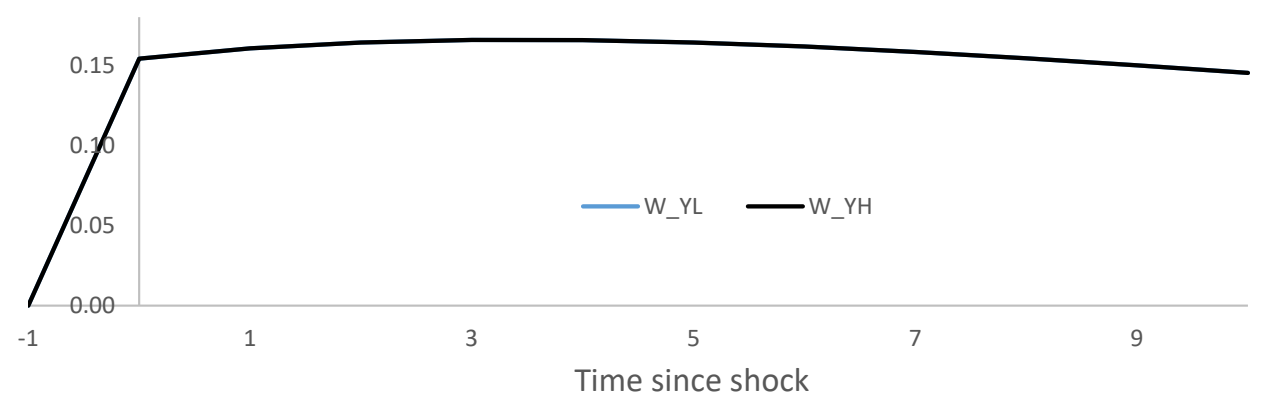

B. Old agents with low value of leisure

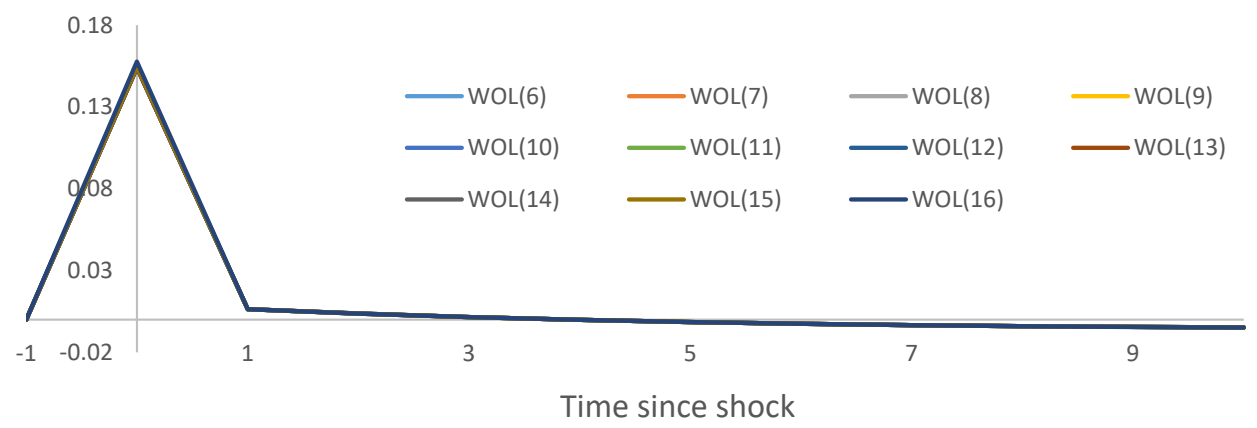

C. Old agents with high value of leisure

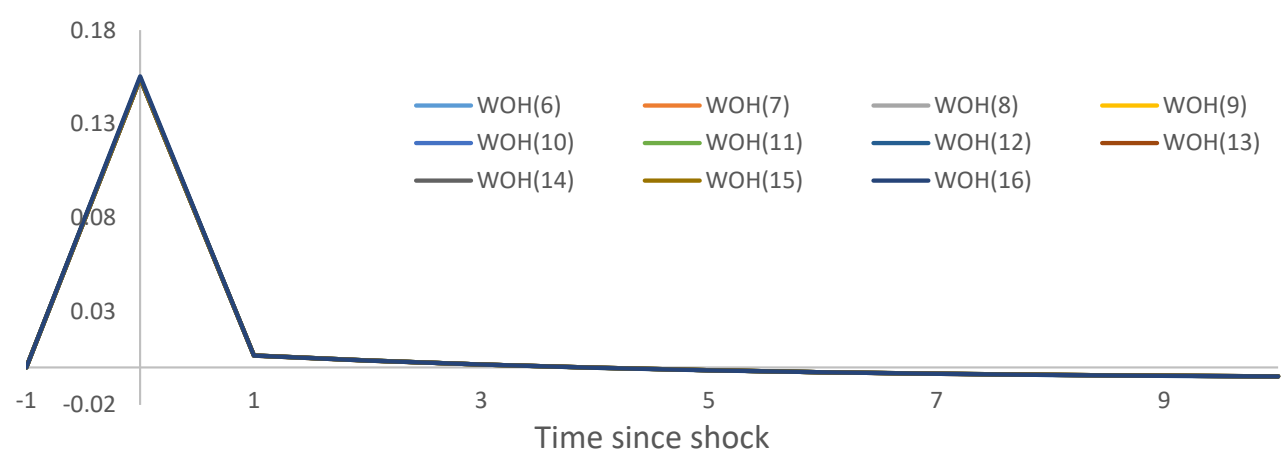


Figure 6: Cross-sectional heterogeneity

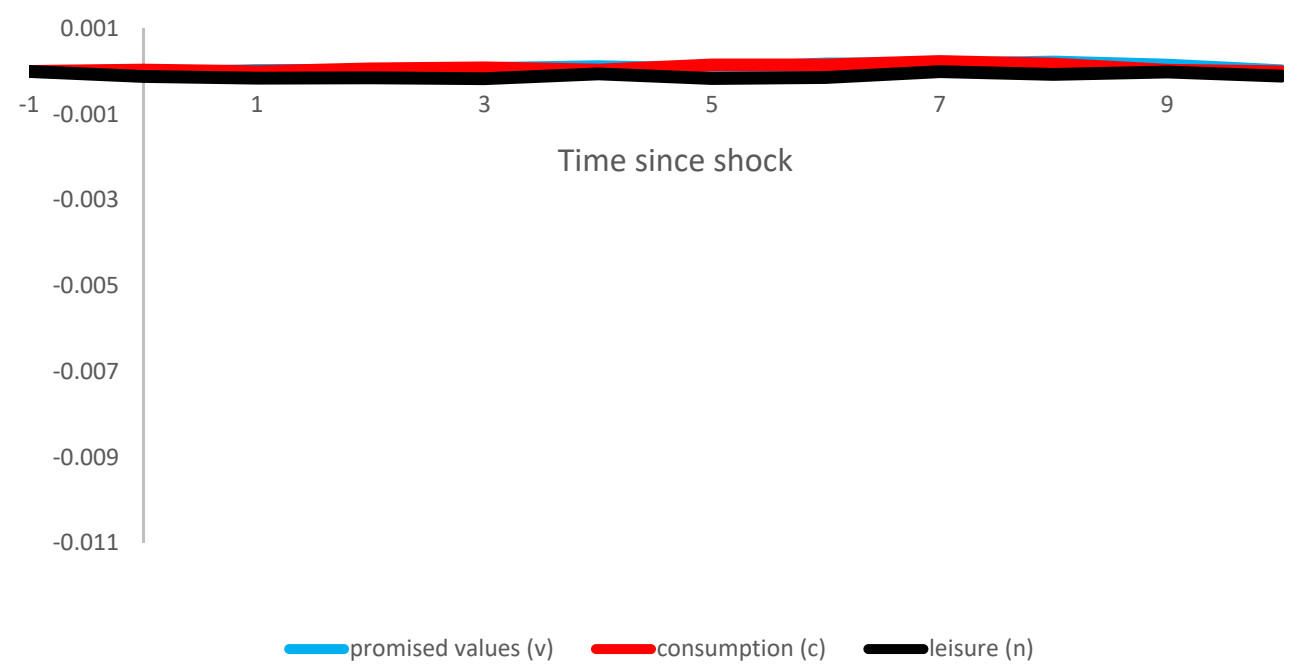


Figure 7: Macro variables

A. Private information

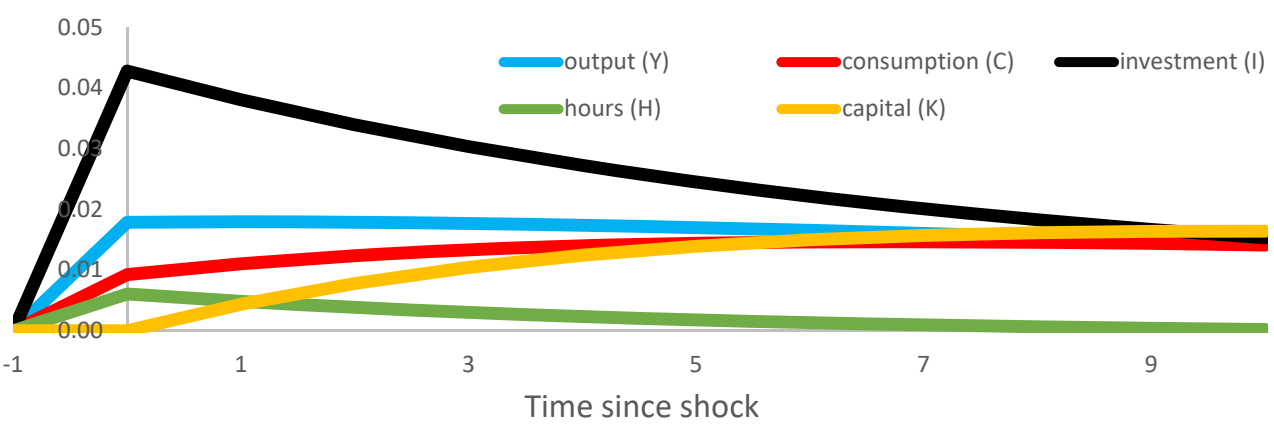

B. Representative agent

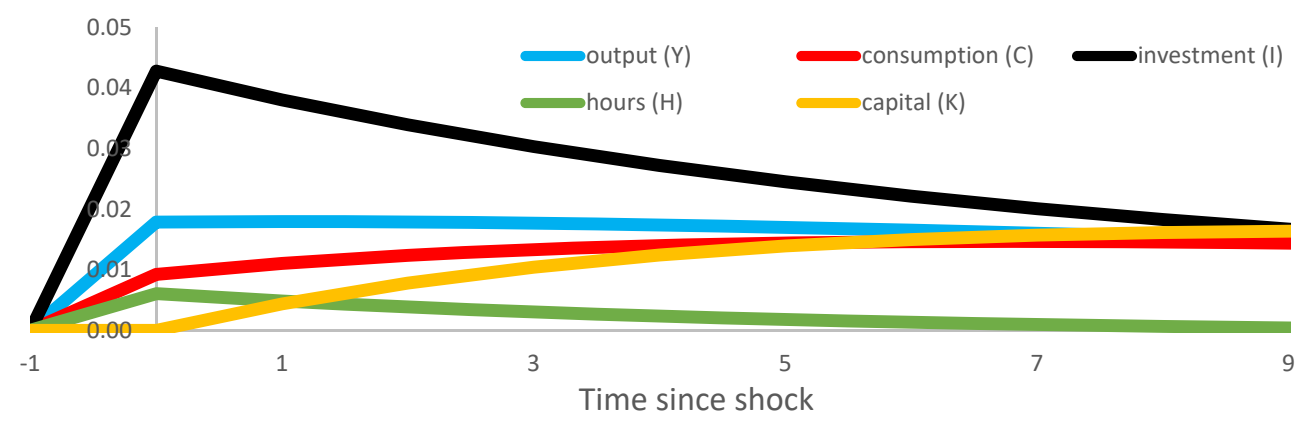

C. Differences

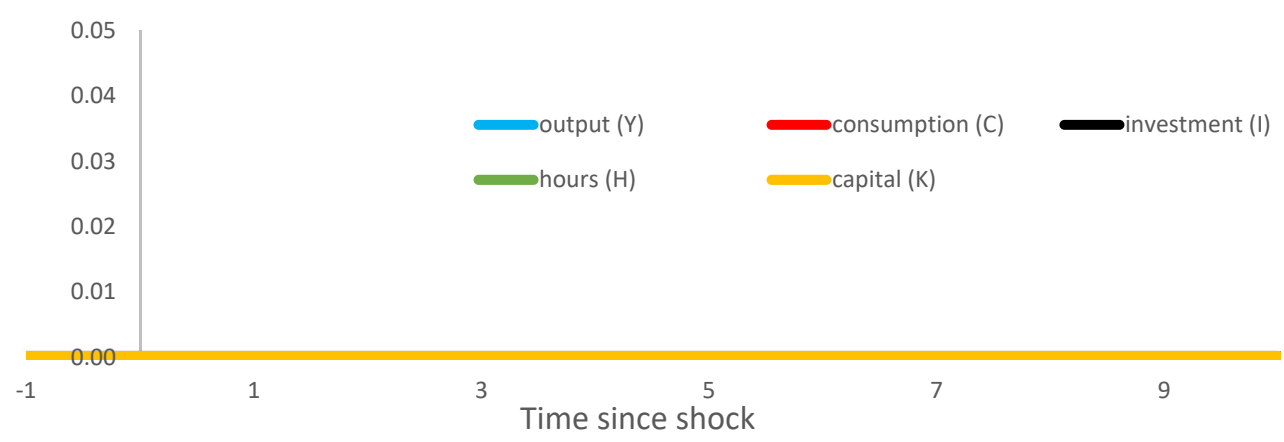


Technical Appendices for

\title{
Computing Equilibria of Stochastic Heterogeneous Agent Models Using Decision Rule Histories
}

\author{
Marcelo Veracierto \\ Federal Reserve Bank of Chicago \\ January, 2020 \\ NOT FOR PUBLICATION
}

This document contains the following appendices:

Technical Appendix 6: Proof of Proposition 1.

Technical Appendix 7: Linearization of the Mirrlees RBC economy 


\section{Proof of Proposition 1}

The deterministic version of equations (2.23)-(2.28) is given by:

$$
\begin{aligned}
0 & =B_{11} x_{t}^{1}+B_{12} x_{t-1}^{2}+C_{12} y_{t}^{2}+D_{1} z_{t} \\
0 & =A_{21} x_{t+1}^{1}+B_{21} x_{t}^{1}+B_{22} x_{t-1}^{2}+C_{21} y_{t+1}^{1} \\
0 & =A_{32} x_{t}^{2}+B_{31} x_{t}^{1}+B_{32} x_{t-1}^{2}+C_{32} y_{t}^{2} \\
0 & =H_{41} x_{t}^{1}+H_{42} x_{t-1}^{2}+J_{42} y_{t+1}^{2}+K_{41} y_{t+1}^{1}+K_{42} y_{t}^{2}+M_{4} z_{t} \\
0 & =F_{52} x_{t+1}^{2}+G_{52} x_{t}^{2}+H_{51} x_{t}^{1}+H_{52} x_{t-1}^{2}+J_{52} y_{t+1}^{2}+K_{51} y_{t+1}^{1}+K_{52} y_{t}^{2}+L_{5} z_{t+1}+M_{5} z_{k}(6.5) \\
z_{t+1} & =N z_{t}
\end{aligned}
$$

The following Lemmas is used in the proof of Proposition 1.

Lemma 2 : Suppose that equations (2.33)-(2.36) are the recursive solution to equations (6.1)(6.6). Then,

$$
\begin{aligned}
P_{11} & =-A_{21}^{-1} B_{21}-A_{21}^{-1} C_{21} R_{11} \\
P_{12} & =-A_{21}^{-1} B_{22}-A_{21}^{-1} C_{21} R_{12} \\
Q_{1} & =-A_{21}^{-1} C_{21} S_{1} \\
R_{11} & =-K_{41}^{-1} H_{41}-K_{41}^{-1} J_{42} R_{21} P_{11}-K_{41}^{-1} J_{42} R_{22} P_{21}-K_{41}^{-1} K_{42} R_{21} \\
R_{12} & =-K_{41}^{-1} H_{42}-K_{41}^{-1} J_{42} R_{21} P_{12}-K_{41}^{-1} J_{42} R_{22} P_{22}-K_{41}^{-1} K_{42} R_{22} \\
S_{1} & =-K_{41}^{-1} J_{42} R_{21} Q_{1}-K_{41}^{-1} J_{42} R_{22} Q_{2}-K_{41}^{-1} J_{42} S_{2} N-K_{41}^{-1} K_{42} S_{2}-K_{41}^{-1} M_{4}
\end{aligned}
$$

Also,

$$
\begin{aligned}
0= & {\left[F_{52} P_{21} P_{11}+F_{52} P_{22} P_{21}+G_{52} P_{21}+H_{51}+J_{52} R_{21} P_{11}+J_{52} R_{22} P_{21}+K_{51} R_{11}+K_{52} R_{21}\right] x_{t}^{1} } \\
& +\left[F_{52} P_{21} P_{12}+F_{52} P_{22} P_{22}+G_{52} P_{22}+H_{52}+J_{52} R_{21} P_{12}+J_{52} R_{22} P_{22}+K_{51} R_{12}+K_{52} R_{22}\right] x_{t-1}^{2} \\
& +\left[F_{52} P_{21} Q_{1}+F_{52} P_{22} Q_{2}+F_{52} Q_{2} N+G_{52} Q_{2}+J_{52} R_{21} Q_{1}+J_{52} R_{22} Q_{2}\right. \\
& \left.+J_{52} S_{2} N+K_{51} S_{1}+K_{52} S_{2}+L_{5} N+M_{5}\right] z_{t}
\end{aligned}
$$


Proof: From equation (6.2) we have that

$$
\begin{aligned}
x_{t+1}^{1} & =-A_{21}^{-1} B_{21} x_{t}^{1}-A_{21}^{-1} B_{22} x_{t-1}^{2}-A_{21}^{-1} C_{21} y_{t+1}^{1} \\
& =-A_{21}^{-1} B_{21} x_{t}^{1}-A_{21}^{-1} B_{22} x_{t-1}^{2}-A_{21}^{-1} C_{21}\left[R_{11} x_{t}^{1}+R_{12} x_{t-1}^{2}+S_{1} z_{t}\right] \\
& =-A_{21}^{-1} B_{21} x_{t}^{1}-A_{21}^{-1} B_{22} x_{t-1}^{2}-A_{21}^{-1} C_{21} R_{11} x_{t}^{1}-A_{21}^{-1} C_{21} R_{12} x_{t-1}^{2}-A_{21}^{-1} C_{21} S_{1} z_{t} \\
& =\left[-A_{21}^{-1} B_{21}-A_{21}^{-1} C_{21} R_{11}\right] x_{t}^{1}+\left[-A_{21}^{-1} B_{22}-A_{21}^{-1} C_{21} R_{12}\right] x_{t-1}^{2}+\left[-A_{21}^{-1} C_{21} S_{1}\right] z_{t}
\end{aligned}
$$

where the second equality uses equation (2.35). Equating coefficients with equation (2.33) gives equations (6.7)-(6.9).

From equation (6.4) we have that

$$
\begin{aligned}
& y_{t+1}^{1}=-K_{41}^{-1} H_{41} x_{t}^{1}-K_{41}^{-1} H_{42} x_{t-1}^{2}-K_{41}^{-1} J_{42} y_{t+1}^{2}-K_{41}^{-1} K_{42} y_{t}^{2}-K_{41}^{-1} M_{4} z_{t} \\
& =-K_{41}^{-1} H_{41} x_{t}^{1}-K_{41}^{-1} H_{42} x_{t-1}^{2}-K_{41}^{-1} J_{42}\left[R_{21} x_{t+1}^{1}+R_{22} x_{t}^{2}+S_{2} z_{t+1}\right] \\
& -K_{41}^{-1} K_{42}\left[R_{21} x_{t}^{1}+R_{22} x_{t-1}^{2}+S_{2} z_{t}\right]-K_{41}^{-1} M_{4} z_{t} \\
& =-K_{41}^{-1} H_{41} x_{t}^{1}-K_{41}^{-1} H_{42} x_{t-1}^{2}-K_{41}^{-1} J_{42} R_{21} x_{t+1}^{1}-K_{41}^{-1} J_{42} R_{22} x_{t}^{2}-K_{41}^{-1} J_{42} S_{2} N z_{t} \\
& -K_{41}^{-1} K_{42} R_{21} x_{t}^{1}-K_{41}^{-1} K_{42} R_{22} x_{t-1}^{2}-K_{41}^{-1} K_{42} S_{2} z_{t}-K_{41}^{-1} M_{4} z_{t} \\
& =-K_{41}^{-1} H_{41} x_{t}^{1}-K_{41}^{-1} H_{42} x_{t-1}^{2}-K_{41}^{-1} J_{42} R_{21}\left[P_{11} x_{t}^{1}+P_{12} x_{t-1}^{2}+Q_{1} z_{t}\right] \\
& -K_{41}^{-1} J_{42} R_{22}\left[P_{21} x_{t}^{1}+P_{22} x_{t-1}^{2}+Q_{2} z_{t}\right]-K_{41}^{-1} J_{42} S_{2} N z_{t} \\
& -K_{41}^{-1} K_{42} R_{21} x_{t}^{1}-K_{41}^{-1} K_{42} R_{22} x_{t-1}^{2}-K_{41}^{-1} K_{42} S_{2} z_{t}-K_{41}^{-1} M_{4} z_{t} \\
& =-K_{41}^{-1} H_{41} x_{t}^{1}-K_{41}^{-1} H_{42} x_{t-1}^{2}-K_{41}^{-1} J_{42} R_{21} P_{11} x_{t}^{1}-K_{41}^{-1} J_{42} R_{21} P_{12} x_{t-1}^{2}-K_{41}^{-1} J_{42} R_{21} Q_{1} z_{t} \\
& -K_{41}^{-1} J_{42} R_{22} P_{21} x_{t}^{1}-K_{41}^{-1} J_{42} R_{22} P_{22} x_{t-1}^{2}-K_{41}^{-1} J_{42} R_{22} Q_{2} z_{t}-K_{41}^{-1} J_{42} S_{2} N z_{t} \\
& -K_{41}^{-1} K_{42} R_{21} x_{t}^{1}-K_{41}^{-1} K_{42} R_{22} x_{t-1}^{2}-K_{41}^{-1} K_{42} S_{2} z_{t}-K_{41}^{-1} M_{4} z_{t} \\
& =\left[-K_{41}^{-1} H_{41}-K_{41}^{-1} J_{42} R_{21} P_{11}-K_{41}^{-1} J_{42} R_{22} P_{21}-K_{41}^{-1} K_{42} R_{21}\right] x_{t}^{1} \\
& +\left[-K_{41}^{-1} H_{42}-K_{41}^{-1} J_{42} R_{21} P_{12}-K_{41}^{-1} J_{42} R_{22} P_{22}-K_{41}^{-1} K_{42} R_{22}\right] x_{t-1}^{2} \\
& +\left[-K_{41}^{-1} J_{42} R_{21} Q_{1}-K_{41}^{-1} J_{42} R_{22} Q_{2}-K_{41}^{-1} J_{42} S_{2} N-K_{41}^{-1} K_{42} S_{2}-K_{41}^{-1} M_{4}\right] z_{t}
\end{aligned}
$$

where the second equality uses equation (2.36), the third equality uses equation (6.6), and the fourth equality uses equations (2.33) and (2.34). Equating coefficients with equation (2.35) gives equations (6.10)-(6.12). 
Finally, from equations (2.36), (2.33), (2.34), and (6.6) we have

$$
\begin{aligned}
y_{t+1}^{2} & =R_{21} x_{t+1}^{1}+R_{22} x_{t}^{2}+S_{2} z_{t+1} \\
& =R_{21}\left[P_{11} x_{t}^{1}+P_{12} x_{t-1}^{2}+Q_{1} z_{t}\right]+R_{22}\left[P_{21} x_{t}^{1}+P_{22} x_{t-1}^{2}+Q_{2} z_{t}\right]+S_{2} N z_{t} \\
& =R_{21} P_{11} x_{t}^{1}+R_{21} P_{12} x_{t-1}^{2}+R_{21} Q_{1} z_{t}+R_{22} P_{21} x_{t}^{1}+R_{22} P_{22} x_{t-1}^{2}+R_{22} Q_{2} z_{t}+S_{2} N z_{t} \\
& =\left[R_{21} P_{11}+R_{22} P_{21}\right] x_{t}^{1}+\left[R_{21} P_{12}+R_{22} P_{22}\right] x_{t-1}^{2}+\left[R_{21} Q_{1}+R_{22} Q_{2}+S_{2} N\right] z_{t}
\end{aligned}
$$

From equations (6.5), (6.15), (2.33), (2.34), (2.35) and (2.36) we then have

$$
\begin{aligned}
& 0=F_{52} x_{t+1}^{2}+G_{52} x_{t}^{2}+H_{51} x_{t}^{1}+H_{52} x_{t-1}^{2}+J_{52} y_{t+1}^{2}+K_{51} y_{t+1}^{1}+K_{52} y_{t}^{2}+L_{5} z_{t+1}+M_{5} z_{t} \\
& =F_{52} x_{t+1}^{2}+G_{52} x_{t}^{2}+H_{51} x_{t}^{1}+H_{52} x_{t-1}^{2}+J_{52}\left[R_{21} x_{t+1}^{1}+R_{22} x_{t}^{2}+S_{2} z_{t+1}\right] \\
& +K_{51} y_{t+1}^{1}+K_{52} y_{t}^{2}+L_{5} z_{t+1}+M_{5} z_{t} \\
& =F_{52}\left[P_{21} x_{t+1}^{1}+P_{22} x_{t}^{2}+Q_{2} z_{t+1}\right]+G_{52}\left[P_{21} x_{t}^{1}+P_{22} x_{t-1}^{2}+Q_{2} z_{t}\right]+H_{51} x_{t}^{1}+H_{52} x_{t-1}^{2} \\
& +J_{52} R_{21}\left[P_{11} x_{t}^{1}+P_{12} x_{t-1}^{2}+Q_{1} z_{t}\right]+J_{52} R_{22}\left[P_{21} x_{t}^{1}+P_{22} x_{t-1}^{2}+Q_{2} z_{t}\right] \\
& +J_{52} S_{2} N z_{t}+K_{51} y_{t+1}^{1}+K_{52} y_{t}^{2}+L_{5} z_{t+1}+M_{5} z_{t} \\
& =F_{52} P_{21}\left[P_{11} x_{t}^{1}+P_{12} x_{t-1}^{2}+Q_{1} z_{t}\right]+F_{52} P_{22}\left[P_{21} x_{t}^{1}+P_{22} x_{t-1}^{2}+Q_{2} z_{t}\right]+F_{52} Q_{2} N z_{t} \\
& +G_{52} P_{21} x_{t}^{1}+G_{52} P_{22} x_{t-1}^{2}+G_{52} Q_{2} z_{t}+H_{51} x_{t}^{1}+H_{52} x_{t-1}^{2}+J_{52}\left[R_{21} P_{11}+R_{22} P_{21}\right] x_{t}^{1} \\
& +J_{52}\left[R_{21} P_{12}+R_{22} P_{22}\right] x_{t-1}^{2}+J_{52}\left[R_{21} Q_{1}+R_{22} Q_{2}+S_{2} N\right] z_{t} \\
& +K_{51}\left[R_{11} x_{t}^{1}+R_{12} x_{t-1}^{2}+S_{1} z_{t}\right]+K_{52}\left[R_{21} x_{t}^{1}+R_{22} x_{t-1}^{2}+S_{2} z_{t}\right]+L_{5} z_{t+1}+M_{5} z_{t} \\
& =F_{52} P_{21} P_{11} x_{t}^{1}+F_{52} P_{21} P_{12} x_{t-1}^{2}+F_{52} P_{21} Q_{1} z_{t}+F_{52} P_{22} P_{21} x_{t}^{1}+F_{52} P_{22} P_{22} x_{t-1}^{2}+F_{52} P_{22} Q_{2} z_{t} \\
& +F_{52} Q_{2} N z_{t}+G_{52} P_{21} x_{t}^{1}+G_{52} P_{22} x_{t-1}^{2}+G_{52} Q_{2} z_{t}+H_{51} x_{t}^{1}+H_{52} x_{t-1}^{2}+J_{52}\left[R_{21} P_{11}+R_{22} P_{21}\right] x_{t}^{1} \\
& +J_{52}\left[R_{21} P_{12}+R_{22} P_{22}\right] x_{t-1}^{2}+J_{52}\left[R_{21} Q_{1}+R_{22} Q_{2}+S_{2} N\right] z_{t} \\
& +K_{51}\left[R_{11} x_{t}^{1}+R_{12} x_{t-1}^{2}+S_{1} z_{t}\right]+K_{52}\left[R_{21} x_{t}^{1}+R_{22} x_{t-1}^{2}+S_{2} z_{t}\right]+L_{5} z_{t+1}+M_{5} z_{t} \\
& =\left[F_{52} P_{21} P_{11}+F_{52} P_{22} P_{21}+G_{52} P_{21}+H_{51}+J_{52} R_{21} P_{11}+J_{52} R_{22} P_{21}+K_{51} R_{11}+K_{52} R_{21}\right] x_{t}^{1} \\
& +\left[F_{52} P_{21} P_{12}+F_{52} P_{22} P_{22}+G_{52} P_{22}+H_{52}+J_{52} R_{21} P_{12}+J_{52} R_{22} P_{22}+K_{51} R_{12}+K_{52} R_{22}\right] x_{t-1}^{2} \\
& +\left[F_{52} P_{21} Q_{1}+F_{52} P_{22} Q_{2}+F_{52} Q_{2} N+G_{52} Q_{2}+J_{52} R_{21} Q_{1}+J_{52} R_{22} Q_{2}\right. \\
& \left.+J_{52} S_{2} N+K_{51} S_{1}+K_{52} S_{2}+L_{5} N+M_{5}\right] z_{t}
\end{aligned}
$$

Thus, equation (6.13) is satisfied.

Proof of Proposition 1: By assumption, equations (6.1) and (6.3) are satisfied by equations 
(2.34) and (2.36). Since $\Omega_{21}=P_{21}, \Omega_{22}=P_{22}, \Psi_{2}=Q_{2}, \Phi_{21}=R_{21}, \Phi_{22}=R_{22}, \Gamma_{2}=S_{2}$, equations (2.23) and (2.25) are then satisfied by equations (2.30) and (2.32).

Observe that equation (2.24) evaluates as follows:

$$
\begin{aligned}
& A_{21} x_{t+1}^{1}+B_{21} x_{t}^{1}+B_{22} x_{t-1}^{2}+C_{21} y_{t+1}^{1} \\
= & A_{21}\left[\Omega_{11} x_{t}^{1}+\Omega_{12} x_{t-1}^{2}+\Psi_{1} z_{t}+\Theta_{1} z_{t+1}\right]+B_{21} x_{t}^{1}+B_{22} x_{t-1}^{2} \\
& +C_{21}\left[\Phi_{11} x_{t}^{1}+\Phi_{12} x_{t-1}^{2}+\Gamma_{1} z_{t}+\Lambda_{1} z_{t+1}\right] \\
= & A_{21} P_{11} x_{t}^{1}+A_{21} P_{12} x_{t-1}^{2}+A_{21} \Psi_{1} z_{t}+A_{21} \Theta_{1} z_{t+1}+B_{21} x_{t}^{1}+B_{22} x_{t-1}^{2} \\
& +C_{21} R_{11} x_{t}^{1}+C_{21} R_{12} x_{t-1}^{2}+C_{21} \Gamma_{1} z_{t}+C_{21} \Lambda_{1} z_{t+1} \\
= & {\left[A_{21} P_{11}+B_{21}+C_{21} R_{11}\right] x_{t}^{1}+\left[A_{21} P_{12}+B_{22}+C_{21} R_{12}\right] x_{t-1}^{2} } \\
& +\left[A_{21} \Psi_{1}+C_{21} \Gamma_{1}\right] z_{t}+\left[A_{21} \Theta_{1}+C_{21} \Lambda_{1}\right]\left[N z_{t}+\varepsilon_{t+1}\right] \\
= & {\left[A_{21} P_{11}+B_{21}+C_{21} R_{11}\right] x_{t}^{1}+\left[A_{21} P_{12}+B_{22}+C_{21} R_{12}\right] x_{t-1}^{2} } \\
& +\left[A_{21} \Psi_{1}+C_{21} \Gamma_{1}+A_{21} \Theta_{1} N+C_{21} \Lambda_{1} N\right] z_{t}+\left[A_{21} \Theta_{1}+C_{21} \Lambda_{1}\right] \varepsilon_{t+1} \\
= & {\left[-B_{21}-C_{21} R_{11}+B_{21}+C_{21} R_{11}\right] x_{t}^{1}+\left[-B_{22}-C_{21} R_{12}+B_{22}+C_{21} R_{12}\right] x_{t-1}^{2} } \\
& +\left[A_{21} \Psi_{1}+C_{21} \Gamma_{1}+A_{21} \Theta_{1} N+C_{21} \Lambda_{1} N\right] z_{t}+\left[A_{21} \Theta_{1}+C_{21} \Lambda_{1}\right] \varepsilon_{t+1} \\
= & {\left[A_{21} \Psi_{1}+C_{21} \Gamma_{1}+A_{21} \Theta_{1} N+C_{21} \Lambda_{1} N\right] z_{t}+\left[A_{21} \Theta_{1}+C_{21} \Lambda_{1}\right] \varepsilon_{t+1} }
\end{aligned}
$$

where the first equality uses equations (2.29) and (2.31), the second equality uses the fact that $\Omega_{11}=P_{11}, \Omega_{12}, \Phi_{11}=R_{11}, \Phi_{12}=R_{12}, \Phi_{21}=R_{21}, \Phi_{22}=R_{22}$, and $\Gamma_{2}=S_{2}$, the third equality uses equation (2.28), and the fifth equality uses equations (6.7) and (6.8).

Observe that

$$
\begin{aligned}
A_{21} \Theta_{1}+C_{21} \Lambda_{1}= & A_{21} \Theta_{1}-C_{21} K_{41}^{-1} J_{42} R_{21} \Theta_{1}-C_{21} K_{41}^{-1} J_{42} S_{2} \\
= & A_{21}\left[I-A_{21}^{-1} C_{21} K_{41}^{-1} J_{42} R_{21}\right] \Theta_{1}-C_{21} K_{41}^{-1} J_{42} S_{2} \\
= & A_{21}\left[I-A_{21}^{-1} C_{21} K_{41}^{-1} J_{42} R_{21}\right]\left[I-A_{21}^{-1} C_{21} K_{41}^{-1} J_{42} R_{21}\right]^{-1} A_{21}^{-1} C_{21} K_{41}^{-1} J_{42} S_{2} \\
& -C_{21} K_{41}^{-1} J_{42} S_{2} \\
= & A_{21} A_{21}^{-1} C_{21} K_{41}^{-1} J_{42} S_{2}-C_{21} K_{41}^{-1} J_{42} S_{2} \\
= & C_{21} K_{41}^{-1} J_{42} S_{2}-C_{21} K_{41}^{-1} J_{42} S_{2} \\
= & 0
\end{aligned}
$$

where the first equality uses equation (2.39), and the third equality uses equations (2.37) and 
(2.41).

Also observe that

$$
\begin{aligned}
& A_{21} \Psi_{1}+C_{21} \Gamma_{1}+A_{21} \Theta_{1} N+C_{21} \Lambda_{1} N \\
= & A_{21} \Psi_{1}+C_{21} \Gamma_{1} \\
= & A_{21} \Psi_{1}-C_{21} K_{41}^{-1} J_{42} R_{21} \Psi_{1}-C_{21} K_{41}^{-1} J_{42} R_{22} Q_{2}-C_{21} K_{41}^{-1} K_{42} S_{2}-C_{21} K_{41}^{-1} M_{4} \\
= & A_{21}\left[I-A_{21}^{-1} C_{21} K_{41}^{-1} J_{42} R_{21}\right] \Psi_{1}-C_{21} K_{41}^{-1} J_{42} R_{22} Q_{2}-C_{21} K_{41}^{-1} K_{42} S_{2}-C_{21} K_{41}^{-1} M_{4} \\
= & A_{21}\left[I-A_{21}^{-1} C_{21} K_{41}^{-1} J_{42} R_{21}\right] \Upsilon\left[A_{21}^{-1} C_{21} K_{41}^{-1} J_{42} R_{22} Q_{2}+A_{21}^{-1} C_{21} K_{41}^{-1} K_{42} S_{2}+A_{21}^{-1} C_{21} K_{41}^{-1} M_{4}\right] \\
& -C_{21} K_{41}^{-1} J_{42} R_{22} Q_{2}-C_{21} K_{41}^{-1} K_{42} S_{2}-C_{21} K_{41}^{-1} M_{4} \\
= & A_{21}\left[A_{21}^{-1} C_{21} K_{41}^{-1} J_{42} R_{22} Q_{2}+A_{21}^{-1} C_{21} K_{41}^{-1} K_{42} S_{2}+A_{21}^{-1} C_{21} K_{41}^{-1} M_{4}\right] \\
& -C_{21} K_{41}^{-1} J_{42} R_{22} Q_{2}-C_{21} K_{41}^{-1} K_{42} S_{2}-C_{21} K_{41}^{-1} M_{4} \\
= & C_{21} K_{41}^{-1} J_{42} R_{22} Q_{2}+C_{21} K_{41}^{-1} K_{42} S_{2}+C_{21} K_{41}^{-1} M_{4}-C_{21} K_{41}^{-1} J_{42} R_{22} Q_{2} \\
& -C_{21} K_{41}^{-1} K_{42} S_{2}-C_{21} K_{41}^{-1} M_{4} \\
= & 0
\end{aligned}
$$

where the first equality uses equation (6.17), the second equality uses equation (2.40), the fourth equality uses equation (2.38) and the fifth equality uses equation (2.41).

From equations (6.16), (6.17) and (6.18) it follows that equation (2.24) is satisfied. 
Observe that equation (2.26) evaluates as follows:

$$
\begin{aligned}
& H_{41} x_{t}^{1}+H_{42} x_{t-1}^{2}+J_{42} y_{t+1}^{2}+K_{41} y_{t+1}^{1}+K_{42} y_{t}^{2}+M_{4} z_{t} \\
& =H_{41} x_{t}^{1}+H_{42} x_{t-1}^{2}+J_{42}\left[\Phi_{21} x_{t+1}^{1}+\Phi_{22} x_{t}^{2}+\Gamma_{2} z_{t+1}\right]+K_{41}\left[\Phi_{11} x_{t}^{1}+\Phi_{12} x_{t-1}^{2}+\Gamma_{1} z_{t}+\Lambda_{1} z_{t+1}\right] \\
& +K_{42}\left[\Phi_{21} x_{t}^{1}+\Phi_{22} x_{t-1}^{2}+\Gamma_{2} z_{t}\right]+M_{4} z_{t} \\
& =H_{41} x_{t}^{1}+H_{42} x_{t-1}^{2}+J_{42} R_{21} x_{t+1}^{1}+J_{42} R_{22} x_{t}^{2}+J_{42} S_{2} z_{t+1}+K_{41} R_{11} x_{t}^{1}+K_{41} R_{12} x_{t-1}^{2} \\
& +K_{41} \Gamma_{1} z_{t}+K_{41} \Lambda_{1} z_{t+1}+K_{42} R_{21} x_{t}^{1}+K_{42} R_{22} x_{t-1}^{2}+K_{42} S_{2} z_{t}+M_{4} z_{t} \\
& =H_{41} x_{t}^{1}+H_{42} x_{t-1}^{2}+J_{42} R_{21}\left[\Omega_{11} x_{t}^{1}+\Omega_{12} x_{t-1}^{2}+\Psi_{1} z_{t}+\Theta_{1} z_{t+1}\right] \\
& +J_{42} R_{22}\left[\Omega_{21} x_{t}^{1}+\Omega_{22} x_{t-1}^{2}+\Psi_{2} z_{t}\right]+J_{42} S_{2} z_{t+1}+K_{41} R_{11} x_{t}^{1}+K_{41} R_{12} x_{t-1}^{2}+K_{41} \Gamma_{1} z_{t} \\
& +K_{41} \Lambda_{1} z_{t+1}+K_{42} R_{21} x_{t}^{1}+K_{42} R_{22} x_{t-1}^{2}+K_{42} S_{2} z_{t}+M_{4} z_{t} \\
& =H_{41} x_{t}^{1}+H_{42} x_{t-1}^{2}+J_{42} R_{21} P_{11} x_{t}^{1}+J_{42} R_{21} P_{12} x_{t-1}^{2}+J_{42} R_{21} \Psi_{1} z_{t}+J_{42} R_{21} \Theta_{1} z_{t+1} \\
& +J_{42} R_{22} P_{21} x_{t}^{1}+J_{42} R_{22} P_{22} x_{t-1}^{2}+J_{42} R_{22} Q_{2} z_{t}+J_{42} S_{2} z_{t+1}+K_{41} R_{11} x_{t}^{1}+K_{41} R_{12} x_{t-1}^{2} \\
& +K_{41} \Gamma_{1} z_{t}+K_{41} \Lambda_{1} z_{t+1}+K_{42} R_{21} x_{t}^{1}+K_{42} R_{22} x_{t-1}^{2}+K_{42} S_{2} z_{t}+M_{4} z_{t} \\
& =\left[H_{41}+J_{42} R_{21} P_{11}+J_{42} R_{22} P_{21}+K_{41} R_{11}+K_{42} R_{21}\right] x_{t}^{1} \\
& +\left[H_{42}+J_{42} R_{21} P_{12}+J_{42} R_{22} P_{22}+K_{41} R_{12}+K_{42} R_{22}\right] x_{t-1}^{2} \\
& +\left[J_{42} R_{21} \Psi_{1}+J_{42} R_{22} Q_{2}+K_{41} \Gamma_{1}+K_{42} S_{2}+M_{4}\right] z_{t}+\left[J_{42} R_{21} \Theta_{1}+J_{42} S_{2}+K_{41} \Lambda_{1}\right] z_{t+1} \\
& =\left[H_{41}+J_{42} R_{21} P_{11}+J_{42} R_{22} P_{21}+\right. \\
& \left.K_{41}\left(-K_{41}^{-1} H_{41}-K_{41}^{-1} J_{42} R_{21} P_{11}-K_{41}^{-1} J_{42} R_{22} P_{21}-K_{41}^{-1} K_{42} R_{21}\right)+K_{42} R_{21}\right] x_{t}^{1} \\
& +\left[H_{42}+J_{42} R_{21} P_{12}+J_{42} R_{22} P_{22}\right. \\
& \left.+K_{41}\left(-K_{41}^{-1} H_{42}-K_{41}^{-1} J_{42} R_{21} P_{12}-K_{41}^{-1} J_{42} R_{22} P_{22}-K_{41}^{-1} K_{42} R_{22}\right)+K_{42} R_{22}\right] x_{t-1}^{2} \\
& +\left[J_{42} R_{21} \Psi_{1}+J_{42} R_{22} Q_{2}+K_{41} \Gamma_{1}+K_{42} S_{2}+M_{4}\right] z_{t}+\left[J_{42} R_{21} \Theta_{1}+J_{42} S_{2}+K_{41} \Lambda_{1}\right] z_{t+1} \\
& =\left[H_{41}+J_{42} R_{21} P_{11}+J_{42} R_{22} P_{21}-H_{41}-J_{42} R_{21} P_{11}-J_{42} R_{22} P_{21}-K_{42} R_{21}+K_{42} R_{21}\right] x_{t}^{1} \\
& +\left[H_{42}+J_{42} R_{21} P_{12}+J_{42} R_{22} P_{22}-H_{42}-J_{42} R_{21} P_{12}-J_{42} R_{22} P_{22}-K_{42} R_{22}+K_{42} R_{22}\right] x_{t-1}^{2} \\
& +\left[J_{42} R_{21} \Psi_{1}+J_{42} R_{22} Q_{2}+K_{41} \Gamma_{1}+K_{42} S_{2}+M_{4}\right] z_{t}+\left[J_{42} R_{21} \Theta_{1}+J_{42} S_{2}+K_{41} \Lambda_{1}\right] z_{t+1} \\
& =\left[J_{42} R_{21} \Psi_{1}+J_{42} R_{22} Q_{2}+K_{41} \Gamma_{1}+K_{42} S_{2}+M_{4}\right] z_{t}+\left[J_{42} R_{21} \Theta_{1}+J_{42} S_{2}+K_{41} \Lambda_{1}\right] z_{t+1}
\end{aligned}
$$


$\Psi_{2}=Q_{2}$, where the sixth equality uses equations (6.10) and (6.11),

Observe that

$$
\begin{aligned}
J_{42} R_{21} \Theta_{1}+J_{42} S_{2}+K_{41} \Lambda_{1} & =J_{42} R_{21} \Theta_{1}+J_{42} S_{2}-J_{42} R_{21} \Theta_{1}-J_{42} S_{2} \\
& =0
\end{aligned}
$$

where the first equality uses equation (2.39).

Also observe that

$$
\begin{aligned}
& J_{42} R_{21} \Psi_{1}+J_{42} R_{22} Q_{2}+K_{41} \Gamma_{1}+K_{42} S_{2}+M_{4} \\
= & J_{42} R_{21} \Psi_{1}+J_{42} R_{22} Q_{2}-J_{42} R_{21} \Psi_{1}-J_{42} R_{22} Q_{2}-K_{42} S_{2}-M_{4}+K_{42} S_{2}+M_{4} \\
= & 0
\end{aligned}
$$

where the first equality uses equation (2.40).

From equations (6.19), (6.20) and (6.21) it follows that equation (2.26) is satisfied.

It remains to show that equation (2.27) holds.

Applying conditional expectations to equations (2.29)-(2.32) and using the fact that $\Omega_{11}=P_{11}$, $\Omega_{12}=P_{12}, \Omega_{21}=P_{21}, \Omega_{22}=P_{22}, \Psi_{2}=Q_{2}, \Phi_{11}=R_{11}, \Phi_{12}=R_{12}, \Phi_{21}=R_{21}, \Phi_{22}=R_{22}, \Gamma_{2}=S_{2}$, we have

$$
\begin{aligned}
E_{t}\left(x_{t+1}^{1}\right) & =P_{11} x_{t}^{1}+P_{12} x_{t-1}^{2}+\left[\Psi_{1}+\Theta_{1} N\right] z_{t}, \\
E_{t}\left(x_{t}^{2}\right) & =P_{21} x_{t}^{1}+P_{22} x_{t-1}^{2}+Q_{2} z_{t}, \\
E_{t}\left(y_{t+1}^{1}\right) & =R_{11} x_{t}^{1}+R_{12} x_{t-1}^{2}+\left[\Gamma_{1}+\Lambda_{1} N\right] z_{t}, \\
E_{t}\left(y_{t}^{2}\right) & =R_{21} x_{t}^{1}+R_{22} x_{t-1}^{2}+S_{2} z_{t} .
\end{aligned}
$$

From equations (6.9) and (6.12) we have that

$$
\begin{aligned}
Q_{1}= & -A_{21}^{-1} C_{21} S_{1} \\
= & A_{21}^{-1} C_{21} K_{41}^{-1} J_{42} R_{21} Q_{1}+A_{21}^{-1} C_{21} K_{41}^{-1} J_{42} R_{22} Q_{2}+A_{21}^{-1} C_{21} K_{41}^{-1} J_{42} S_{2} N \\
& +A_{21}^{-1} C_{21} K_{41}^{-1} K_{42} S_{2}+A_{21}^{-1} C_{21} K_{41}^{-1} M_{4}
\end{aligned}
$$

Hence,

$$
\begin{aligned}
{\left[I-A_{21}^{-1} C_{21} K_{41}^{-1} J_{42} R_{21}\right] Q_{1}=} & A_{21}^{-1} C_{21} K_{41}^{-1} J_{42} R_{22} Q_{2} \\
& +A_{21}^{-1} C_{21} K_{41}^{-1} J_{42} S_{2} N+A_{21}^{-1} C_{21} K_{41}^{-1} K_{42} S_{2}+A_{21}^{-1} C_{21} K_{41}^{-1} M_{4}
\end{aligned}
$$


Using equations (2.41), (2.38) and (2.37) we then get that

$$
\begin{aligned}
Q_{1} & =\Upsilon\left[A_{21}^{-1} C_{21} K_{41}^{-1} J_{42} R_{22} Q_{2}+A_{21}^{-1} C_{21} K_{41}^{-1} J_{42} S_{2} N+A_{21}^{-1} C_{21} K_{41}^{-1} K_{42} S_{2}+A_{21}^{-1} C_{21} K_{41}^{-1} M_{4}\right] \\
& =\Upsilon\left[A_{21}^{-1} C_{21} K_{41}^{-1} J_{42} R_{22} Q_{2}+A_{21}^{-1} C_{21} K_{41}^{-1} K_{42} S_{2}+A_{21}^{-1} C_{21} K_{41}^{-1} M_{4}\right]+\Upsilon A_{21}^{-1} C_{21} K_{41}^{-1} J_{42} S_{2} N \\
& =\Psi_{1}+\Theta_{1} N
\end{aligned}
$$

Also, using equations (2.40), (2.39), (6.26) and (6.12) we have that

$$
\begin{aligned}
& \Gamma_{1}+\Lambda_{1} N \\
= & -K_{41}^{-1} J_{42} R_{21} \Psi_{1}-K_{41}^{-1} J_{42} R_{22} Q_{2}-K_{41}^{-1} K_{42} S_{2}-K_{41}^{-1} M_{4}-K_{41}^{-1} J_{42} R_{21} \Theta_{1} N-K_{41}^{-1} J_{42} S_{2} N \\
= & -K_{41}^{-1} J_{42} R_{21}\left[\Psi_{1}+\Theta_{1} N\right]-K_{41}^{-1} J_{42} R_{22} Q_{2}-K_{41}^{-1} K_{42} S_{2}-K_{41}^{-1} M_{4}-K_{41}^{-1} J_{42} S_{2} N \\
= & S_{1}
\end{aligned}
$$

Using equations (6.26) and (6.27) we can then write equations (6.22)-(6.25) as follows:

$$
\begin{aligned}
E_{t}\left(x_{t+1}^{1}\right) & =P_{11} x_{t}^{1}+P_{12} x_{t-1}^{2}+Q_{1} z_{t}, \\
E_{t}\left(x_{t}^{2}\right) & =P_{21} x_{t}^{1}+P_{22} x_{t-1}^{2}+Q_{2} z_{t} \\
E_{t}\left(y_{t+1}^{1}\right) & =R_{11} x_{t}^{1}+R_{12} x_{t-1}^{2}+S_{1} z_{t} \\
E_{t}\left(y_{t}^{2}\right) & =R_{21} x_{t}^{1}+R_{22} x_{t-1}^{2}+S_{2} z_{t} .
\end{aligned}
$$

From equation (6.31) we have

$$
E_{t+1}\left(y_{t+1}^{2}\right)=R_{21} x_{t+1}^{1}+R_{22} x_{t}^{2}+S_{2} z_{t+1}
$$

Using the Law of Iterated expectations and equations (6.28) and (6.29) we then get

$$
\begin{aligned}
E_{t}\left(y_{t+1}^{2}\right)= & R_{21} E_{t}\left(x_{t+1}^{1}\right)+R_{22} E_{t}\left(x_{t}^{2}\right)+S_{2} E_{t}\left(z_{t+1}\right) \\
= & R_{21}\left[P_{11} x_{t}^{1}+P_{12} x_{t-1}^{2}+Q_{1} z_{t}\right] \\
& +R_{22}\left[P_{21} x_{t}^{1}+P_{22} x_{t-1}^{2}+Q_{2} z_{t}\right]+S_{2} N z_{t}
\end{aligned}
$$

Also, from equation (6.29) we have

$$
E_{t+1}\left(x_{t+1}^{2}\right)=P_{21} x_{t+1}^{1}+P_{22} x_{t}^{2}+Q_{2} z_{t+1}
$$

Using the Law of Iterated expectations and equations (6.28) and (6.29) we then get

$$
\begin{aligned}
E_{t}\left(x_{t+1}^{2}\right)= & P_{21} E_{t}\left(x_{t+1}^{1}\right)+P_{22} E_{t}\left(x_{t}^{2}\right)+Q_{2} E_{t}\left(z_{t+1}\right) \\
= & P_{21}\left[P_{11} x_{t}^{1}+P_{12} x_{t-1}^{2}+Q_{1} z_{t}\right] \\
& +P_{22}\left[P_{21} x_{t}^{1}+P_{22} x_{t-1}^{2}+Q_{2} z_{t}\right]+Q_{2} N z_{t}
\end{aligned}
$$


Observe that equation (2.27) evaluates as follows

$$
\begin{aligned}
& F_{52} E_{t}\left(x_{t+1}^{2}\right)+G_{52} E_{t}\left(x_{t}^{2}\right)+H_{51} x_{t}^{1}+H_{52} x_{t-1}^{2}+J_{52} E_{t}\left(y_{t+1}^{2}\right)+K_{51} E_{t}\left(y_{t+1}^{1}\right)+K_{52} E_{t}\left(y_{t}^{2}\right) \\
& +L_{5} N z_{t}+M_{5} z_{t} \\
& =F_{52} P_{21}\left[P_{11} x_{t}^{1}+P_{12} x_{t-1}^{2}+Q_{1} z_{t}\right]+F_{52} P_{22}\left[P_{21} x_{t}^{1}+P_{22} x_{t-1}^{2}+Q_{2} z_{t}\right]+F_{52} Q_{2} N z_{t} \\
& +G_{52}\left[P_{21} x_{t}^{1}+P_{22} x_{t-1}^{2}+Q_{2} z_{t}\right]+H_{51} x_{t}^{1}+H_{52} x_{t-1}^{2}+J_{52} R_{21}\left[P_{11} x_{t}^{1}+P_{12} x_{t-1}^{2}+Q_{1} z_{t}\right] \\
& +J_{52} R_{22}\left[P_{21} x_{t}^{1}+P_{22} x_{t-1}^{2}+Q_{2} z_{t}\right]+J_{52} S_{2} N z_{t} \\
& +K_{51}\left[R_{11} x_{t}^{1}+R_{12} x_{t-1}^{2}+S_{1} z_{t}\right]+K_{52}\left[R_{21} x_{t}^{1}+R_{22} x_{t-1}^{2}+S_{2} z_{t}\right]+L_{5} N z_{t}+M_{5} z_{t} \\
& =F_{52} P_{21} P_{11} x_{t}^{1}+F_{52} P_{21} P_{12} x_{t-1}^{2}+F_{52} P_{21} Q_{1} z_{t}+F_{52} P_{22} P_{21} x_{t}^{1}+F_{52} P_{22} P_{22} x_{t-1}^{2}+F_{52} P_{22} Q_{2} z_{t} \\
& +F_{52} Q_{2} N z_{t}+G_{52}\left[P_{21} x_{t}^{1}+P_{22} x_{t-1}^{2}+Q_{2} z_{t}\right]+H_{51} x_{t}^{1}+H_{52} x_{t-1}^{2} \\
& +J_{52} R_{21}\left[P_{11} x_{t}^{1}+P_{12} x_{t-1}^{2}+Q_{1} z_{t}\right]+J_{52} R_{22}\left[P_{21} x_{t}^{1}+P_{22} x_{t-1}^{2}+Q_{2} z_{t}\right]+J_{52} S_{2} N z_{t} \\
& +K_{51}\left[R_{11} x_{t}^{1}+R_{12} x_{t-1}^{2}+S_{1} z_{t}\right]+K_{52}\left[R_{21} x_{t}^{1}+R_{22} x_{t-1}^{2}+S_{2} z_{t}\right]+L_{5} N z_{t}+M_{5} z_{t} \\
& =\left[F_{52} P_{21} P_{11}+F_{52} P_{22} P_{21}+G_{52} P_{21}+H_{51}+J_{52} R_{21} P_{11}+J_{52} R_{22} P_{21}+K_{51} R_{11}+K_{52} R_{21}\right] x_{t}^{1} \\
& +\left[F_{52} P_{21} P_{12}+F_{52} P_{22} P_{22}+G_{52} P_{22}+H_{52}+J_{52} R_{21} P_{12}+J_{52} R_{22} P_{22}+K_{51} R_{12}+K_{52} R_{22}\right] x_{t-1}^{2} \\
& +\left[F_{52} P_{21} Q_{1}+F_{52} P_{22} Q_{2}+F_{52} Q_{2} N+G_{52} Q_{2}+J_{52} R_{21} Q_{1}+J_{52} R_{22} Q_{2}\right. \\
& \left.+J_{52} S_{2} N+K_{51} S_{1}+K_{52} S_{2}+L_{5} N+M_{5}\right] z_{t} \\
& =0
\end{aligned}
$$

where the first equality uses equations (6.33), (6.32), (6.30) and (6.31), and the third equality uses equation (6.13). Thus equation (2.27) is satisfied 


\section{Mirrlees economy: Linearization}

This appendix first lists each of the first order conditions for the Mirrlees RBC economy, describes the arguments involved in each first order condition, and states the total number of each first order condition. The appendix then classifies each of the variables involved into the vectors defined by equations (2.19)-(2.22), classifies each first order condition into one of the five types given by equations (2.23)-(2.27), and provides the total number of equations of each type as well as the total number of variables. Finally, the appendix shows how the general linearized model (2.23)(2.28) simplifies in the Mirrlees RBC economy, as well as the corresponding transformation given by equations (2.37)-(2.41).

Similarly to equations (2.19)-(2.22), $J$ denotes the total number of grid points used in the spline approximations. However, given that promised values must lie in a closed interval, the functions $w_{o L t}(v)$ and $w_{o L t}(v)$ may hit the limits of those intervals when $v$ is close to those limits. In what follows, I denote by $J_{1}$ the lowest grid point for which $w_{o L t}\left(\bar{v}_{j}\right)$ is larger than the low limit and $J_{2}$ the largest grid point for which $w_{o L t}\left(\bar{v}_{j}\right)$ is smaller than the high limit. Similarly, I denote by $J_{3}$ the lowest grid point for which $w_{o H t}\left(\bar{v}_{j}\right)$ is larger than the low limit and $J_{4}$ the largest grid point for which $w_{o H t}\left(\bar{v}_{j}\right)$ is smaller than the high limit.

\subsection{First order conditions}

1) Equation

$$
0=\psi_{L}-\lambda_{t}\left[(1-\varphi) u_{y L t}+1\right]^{\frac{1}{1-\varphi}-1} \psi_{L}+\lambda_{t} \xi_{y t}
$$

becomes

$$
0=L_{u_{y L}}\left(\triangle u_{y L, t}, \triangle \ln \xi_{y t}, \triangle \ln \lambda_{t}\right)
$$

Number of equations: 1

2) Equation

$$
0=\psi_{H}-\lambda_{t}\left[(1-\varphi) u_{y H t}+1\right]^{\frac{1}{1-\varphi}-1} \psi_{H}-\lambda_{t} \xi_{y t}
$$

becomes

$$
0=L_{u_{y H}}\left(\triangle u_{y H, t}, \triangle \ln \xi_{y t}, \triangle \ln \lambda_{t}\right)
$$

Number of equations: 1 


\section{3) Equation}

$$
0=\alpha_{L} \psi_{L}-\lambda_{t} q_{t}\left[(1-\pi) n_{y L t}+1\right]^{\frac{1}{1-\pi}-1} \psi_{L}+\lambda_{t} \alpha_{L} \xi_{y t}
$$

becomes

$$
0=L_{n_{y L}}\left(\triangle n_{y L, t}, \triangle \ln \xi_{y t}, \triangle \ln \lambda_{t}, \triangle \ln q_{t}\right)
$$

Number of equations: 1

4) Equation

$$
0=\alpha_{H} \psi_{H}-\lambda_{t} q_{t}\left[(1-\pi) n_{y H t}+1\right]^{\frac{1}{1-\pi}-1} \psi_{H}-\lambda_{t} \alpha_{L} \xi_{y t}
$$

becomes

$$
0=L_{n_{y H}}\left(\triangle n_{y H, t}, \triangle \ln \xi_{y t}, \triangle \ln \lambda_{t}, \triangle \ln q_{t}\right)
$$

Number of equations: 1

5) Equation

$$
0=\beta \sigma \psi_{L}+\lambda_{t} \beta \sigma \xi_{y t}-\theta \lambda_{t+1} \sigma \psi_{L} \eta_{t+1}\left(w_{y L, t+1}\right)
$$

becomes

$$
0=L_{w_{y L}}\left(\triangle \ln \xi_{y t}, \triangle \ln \lambda_{t}, \triangle \ln \lambda_{t+1},\left[\triangle \ln \eta_{t+1}\left(\bar{v}_{j}\right)\right]_{j=1}^{J}, \triangle w_{y L, t+1}\right)
$$

Number of equations: 1

6) Equation

$$
0=\beta \sigma \psi_{H}-\lambda_{t} \beta \sigma \xi_{y t}-\theta \lambda_{t+1} \sigma \psi_{H} \eta_{t+1}\left(w_{y H, t+1}\right)
$$

becomes

$$
0=L_{w_{y H}}\left(\triangle \ln \xi_{y t}, \triangle \ln \lambda_{t}, \triangle \ln \lambda_{t+1},\left[\triangle \ln \eta_{t+1}\left(\bar{v}_{j}\right)\right]_{j=1}^{J}, \triangle w_{y H, t+1}\right)
$$

Number of equations: 1

7) Equation

$$
0=-\left[(1-\varphi) u_{o L t}(v)+1\right]^{\frac{1}{1-\varphi}-1} \psi_{L}+\xi_{o t}(v)+\eta_{t}(v) \psi_{L}
$$

becomes

$$
0=L_{u_{o L}\left(\bar{v}_{i}\right)}\left(\triangle u_{o L, t}\left(\bar{v}_{i}\right), \triangle \ln \xi_{o t}\left(\bar{v}_{i}\right), \triangle \ln \eta_{t}\left(\bar{v}_{i}\right)\right)
$$

for $i=1, \ldots, J$.

Number of equations: $J$

8) Equation

$$
0=-\left[(1-\varphi) u_{o H t}(v)+1\right]^{\frac{1}{1-\varphi}-1} \psi_{H}-\xi_{o t}(v)+\eta_{t}(v) \psi_{H}
$$


becomes

$$
0=L_{u_{o H}\left(\bar{v}_{i}\right)}\left(\triangle u_{o H, t}\left(\bar{v}_{i}\right), \triangle \ln \xi_{o t}\left(\bar{v}_{i}\right), \triangle \ln \eta_{t}\left(\bar{v}_{i}\right)\right)
$$

for $i=1, \ldots, J$.

Number of equations: $J$

9) Equation

$$
0=-q_{t}\left[(1-\pi) n_{o L t}(v)+1\right]^{\frac{1}{1-\pi}-1} \psi_{L}+\alpha_{L} \xi_{o t}(v)+\eta_{t}(v) \alpha_{L} \psi_{L}
$$

becomes

$$
0=L_{n_{o L}\left(\bar{v}_{i}\right)}\left(\triangle n_{o L, t}\left(\bar{v}_{i}\right), \triangle \ln \xi_{o t}\left(\bar{v}_{i}\right), \triangle \ln \eta_{t}\left(\bar{v}_{i}\right), \triangle \ln q_{t}\right)
$$

for $i=1, \ldots, J$.

Number of equations: $J$

10) Equation

$$
0=-q_{t}\left[(1-\pi) n_{o H t}(v)+1\right]^{\frac{1}{1-\pi}-1} \psi_{H}-\alpha_{L} \xi_{o t}(v)+\eta_{t}(v) \alpha_{H} \psi_{H}
$$

becomes

$$
0=L_{n_{o H}\left(\bar{v}_{i}\right)}\left(\triangle n_{o H, t}\left(\bar{v}_{i}\right), \triangle \ln \xi_{o t}\left(\bar{v}_{i}\right), \triangle \ln \eta_{t}\left(\bar{v}_{i}\right), \triangle \ln q_{t}\right)
$$

for $i=1, \ldots, J$.

Number of equations: $J$

11) Equation

$$
0=\lambda_{t} \beta \sigma \xi_{o t}(v)+\lambda_{t} \eta_{t}(v) \beta \sigma \psi_{L}-\theta \lambda_{t+1} \sigma \psi_{L} \eta_{t+1}\left[w_{o L, t+1}(v)\right]
$$

becomes

$$
0=L_{w_{o L}\left(\bar{v}_{i}\right)}\left(\triangle \ln \xi_{o t}\left(\bar{v}_{i}\right), \triangle \ln \lambda_{t}, \triangle \ln \eta_{t}\left(\bar{v}_{i}\right), \triangle \ln \lambda_{t+1},\left[\triangle \ln \eta_{t+1}\left(\bar{v}_{j}\right)\right]_{j=1}^{J}, \triangle w_{o L, t+1}\left(\bar{v}_{i}\right)\right)
$$

for $i=J_{1}, \ldots, J_{2}$.

Number of equations: $J_{2}-J_{1}+1$

12) Equation

$$
0=-\lambda_{t} \beta \sigma \xi_{o t}(v)+\lambda_{t} \eta_{t}(v) \beta \sigma \psi_{H}-\theta \lambda_{t+1} \sigma \psi_{H} \eta_{t+1}\left[w_{o H, t+1}(v)\right]
$$


becomes

$$
0=L_{w_{o H}\left(\bar{v}_{i}\right)}\left(\triangle \ln \xi_{o t}\left(\bar{v}_{i}\right), \triangle \ln \lambda_{t}, \triangle \ln \eta_{t}\left(\bar{v}_{i}\right), \triangle \ln \lambda_{t+1},\left[\triangle \ln \eta_{t+1}\left(\bar{v}_{j}\right)\right]_{j=1}^{J}, \triangle w_{o H, t+1}\left(\bar{v}_{i}\right)\right)
$$

for $i=J_{3}, \ldots, J_{4}$.

Number of equations: $J_{4}-J_{3}+1$

13) Equation

$$
0=u_{y L t}+\alpha_{L} n_{y L t}+\beta \sigma E_{t}\left[w_{y L, t+1}\right]-\left\{u_{y H t}+\alpha_{L} n_{y H t}+\beta \sigma E_{t}\left[w_{y H, t+1}\right]\right\}
$$

becomes

$$
0=E_{t}\left[L_{I C^{y}}\left(\triangle u_{y L, t}, \triangle n_{y L, t}, \triangle w_{y L, t+1}, \triangle u_{y H, t}, \triangle n_{y H, t}, \triangle w_{y H, t+1}\right)\right]
$$

Number of equations: 1

14) Equation

$$
0=u_{o L t}(v)+\alpha_{L} n_{o L t}(v)+\beta \sigma E_{t}\left[w_{o L, t+1}(v)\right]-\left\{u_{o H t}(v)+\alpha_{L} n_{o H t}(v)+\beta \sigma E_{t}\left[w_{o H, t+1}(v)\right]\right\}
$$

becomes

$$
0=E_{t}\left[L_{I C^{o}\left(\bar{v}_{i}\right)}\left(\triangle u_{o L, t}\left(\bar{v}_{i}\right), \triangle n_{o L, t}\left(\bar{v}_{i}\right), \triangle w_{o L, t+1}\left(\bar{v}_{i}\right), \triangle u_{o H, t}\left(\bar{v}_{i}\right), \triangle n_{o H, t}\left(\bar{v}_{i}\right), \triangle w_{o H, t+1}\left(\bar{v}_{i}\right)\right)\right]
$$

for $i=1, \ldots, J$.

Number of equations: $J$

15) Equation

$$
\begin{gathered}
0=v-\left\{u_{o L t}(v)+\alpha_{L} n_{o L t}(v)+\beta \sigma E_{t}\left[w_{o L, t+1}(v)\right]\right\} \psi_{L} \\
-\left\{u_{o H t}(v)+\alpha_{H} n_{o H t}(v)+\beta \sigma E_{t}\left[w_{o H, t+1}(v)\right]\right\} \psi_{H}
\end{gathered}
$$

becomes

$$
0=E_{t}\left[L_{P K^{o}\left(\bar{v}_{i}\right)}\left(\triangle u_{o L, t}\left(\bar{v}_{i}\right), \triangle n_{o L, t}\left(\bar{v}_{i}\right), \triangle w_{o L, t+1}\left(\bar{v}_{i}\right), \triangle u_{o H, t}\left(\bar{v}_{i}\right), \triangle n_{o H, t}\left(\bar{v}_{i}\right), \triangle w_{o H, t+1}\left(\bar{v}_{i}\right)\right)\right]
$$

Number of equations: $J$

16) Equation

$$
0=q_{t}-e^{z_{t}} K_{t-1}^{\gamma}(1-\gamma) H_{t}^{-\gamma}
$$

becomes

$$
0=L_{q}\left(\triangle \ln q_{t}, \triangle \ln K_{t-1}, \triangle \ln H_{t}, \triangle z_{t}\right)
$$


Number of equations: 1

17) Equation

$$
0=-\lambda_{t}+\theta E_{t}\left\{\lambda_{t+1}\left[e^{z_{t+1}} \gamma K_{t}^{\gamma-1} H_{t+1}^{1-\gamma}+1-\delta\right]\right\}
$$

becomes

$$
0=E_{t}\left[L_{\lambda}\left(\triangle \ln \lambda_{t}, \triangle \ln K_{t}, \triangle \ln \lambda_{t+1}, \triangle \ln H_{t+1}, \triangle z_{t+1}\right)\right]
$$

Number of equations: 1

18) Equation

$$
\begin{aligned}
0 & =(1-\sigma)\left[(1-\varphi) u_{y L t}+1\right]^{\frac{1}{1-\varphi}} \psi_{L}+(1-\sigma)\left[(1-\varphi) u_{y H t}+1\right]^{\frac{1}{1-\varphi}} \psi_{H} \\
& +\int\left[(1-\alpha) u_{o L t}(v)+1\right]^{\frac{1}{1-\alpha}} \psi_{L} d \mu_{t}+\int\left[(1-\alpha) u_{o H t}(v)+1\right]^{\frac{1}{1-\alpha}} \psi_{H} d \mu_{t} \\
& +I_{t}-e^{z_{t}} K_{t-1}^{\gamma} H_{t}^{1-\gamma}
\end{aligned}
$$

becomes

$$
\begin{aligned}
0 & =L_{Y}\left(\triangle u_{y L, t}, \triangle u_{y H, t},\left[\triangle u_{o L, t}\left(\bar{v}_{j}\right)\right]_{j=1}^{J},\left[\triangle u_{o H, t}\left(\bar{v}_{j}\right)\right]_{j=1}^{J}, \triangle \ln I_{t}, \triangle \ln K_{t-1},\right. \\
& \left.\triangle \ln H_{t}, \triangle z_{t},\left\{\triangle w_{y L, t-n}, \triangle w_{y H, t-n},\left[\triangle w_{o L, t-n}\left(\bar{v}_{j}\right)\right]_{j=J_{1}}^{J_{2}},\left[\triangle w_{o H, t-n}\left(\bar{v}_{j}\right)\right]_{i=J_{3}}^{J_{4}}\right\}_{n=0}^{N}\right)
\end{aligned}
$$

Observe that this linear approximation must be done numerically using Monte Carlo simulations.

Number of equations: 1

19) Equation

$$
\begin{aligned}
0 & =(1-\sigma)\left\{1-\left[(1-\pi) n_{y L t}+1\right]^{\frac{1}{1-\pi}}\right\} \psi_{L}+(1-\sigma)\left\{1-\left[(1-\pi) n_{y H t}+1\right]^{\frac{1}{1-\pi}}\right\} \psi_{H} \\
& +\int\left\{1-\left[(1-\pi) n_{o L t}(v)+1\right]^{\frac{1}{1-\pi}}\right\} \psi_{L} d \mu_{t}+\int\left\{1-\left[(1-\pi) n_{o H t}(v)+1\right]^{\frac{1}{1-\pi}}\right\} \psi_{H} d \mu_{t}-H_{t}
\end{aligned}
$$

becomes

$$
\begin{gathered}
0=L_{H}\left(\triangle n_{y L, t}, \triangle n_{y H, t},\left[\triangle n_{o L, t}\left(\bar{v}_{j}\right)\right]_{j=1}^{J},\left[\triangle n_{o H, t}\left(\bar{v}_{j}\right)\right]_{j=1}^{J}, \triangle \ln H_{t}\right. \\
\left.\left\{\triangle w_{y L, t-j}, \triangle w_{y H, t-n},\left[\triangle w_{o L, t-n}\left(\bar{v}_{j}\right)\right]_{j=J_{1}}^{J_{2}},\left[\triangle w_{o H, t-n}\left(\bar{v}_{j}\right)\right]_{j=J_{3}}^{J_{4}}\right\}_{n=0}^{N}\right)
\end{gathered}
$$

Observe that this linear approximation also must be done numerically, using Monte Carlo simulations.

Number of equations: 1 
20) Equation

$$
0=K_{t}-(1-\delta) K_{t-1}-I_{t}
$$

becomes

$$
L_{I}\left(\triangle \ln K_{t}, \triangle \ln K_{t-1}, \triangle \ln I_{t}\right)
$$

Number of equations: 1

21) Equation

$$
\begin{aligned}
& {\left[\triangle w_{y L, t+1-n}, \triangle w_{y H, t+1-n},\left[\triangle w_{o L, t+1-n}\left(\bar{v}_{j}\right)\right]_{j=J_{1}}^{J_{2}},\left[\triangle w_{o H, t+1-n}\left(\bar{v}_{j}\right)\right]_{j=J_{3}}^{J_{4}}\right]_{n=0}^{N}} \\
& =\triangle w_{y L, t+1}, \triangle w_{y H, t+1},\left[\triangle w_{o L, t+1}\left(\bar{v}_{j}\right)\right]_{j=J_{1}}^{J_{2}},\left[\triangle w_{o H, t+1}\left(\bar{v}_{j}\right)\right]_{j=J_{3}}^{J_{4}}, \\
& {\left[\triangle w_{y L, t-n}, \triangle w_{y H, t-n},\left[\triangle w_{o L, t-n}\left(\bar{v}_{j}\right)\right]_{j=J_{1}}^{J_{2}},\left[\triangle w_{o H, t-n}\left(\bar{v}_{j}\right)\right]_{j=J_{3}}^{J_{4}}\right]_{n=0}^{N-1}}
\end{aligned}
$$

becomes

$$
0=L_{\mu}\left(\begin{array}{c}
\triangle w_{y L, t+1}, \triangle w_{y H, t+1},\left[\triangle w_{o L, t+1}\left(\bar{v}_{j}\right)\right]_{j=J_{1}}^{J_{2}},\left[\triangle w_{o H, t+1}\left(\bar{v}_{j}\right)\right]_{j=J_{3}}^{J_{4}}, \\
{\left[\triangle w_{y L, t-n}, \triangle w_{y H, t-n},\left[\triangle w_{o L, t-n}\left(\bar{v}_{j}\right)\right]_{j=J_{1}}^{J_{2}},\left[\triangle w_{o H, t-n}\left(\bar{v}_{j}\right)\right]_{j=J_{3}}^{J_{4}}\right]_{n=0}^{N-1}}
\end{array}\right)
$$

Number of equations: $(N+1)\left[2+\left(J_{2}-J_{1}+1\right)+\left(J_{4}-J_{3}+1\right)\right]$

\subsection{Classification of variables and equations}

Classify the variables as follows:

$$
\begin{gathered}
x_{t}^{1}=\left\{\triangle w_{y L, t-n}, \triangle w_{y H, t-n},\left[\triangle w_{o L, t-n}\left(\bar{v}_{j}\right)\right]_{j=J_{1}}^{J_{2}},\left[\triangle w_{o H, t-n}\left(\bar{v}_{j}\right)\right]_{j=J_{3}}^{J_{4}}\right\}_{n=0}^{N} \\
x_{t-1}^{2}=\left\{\triangle \ln K_{t-1}\right\} \\
y_{t+1}^{1}=\left\{\triangle w_{y L, t+1}, \triangle w_{y H, t+1},\left[\triangle w_{o L, t+1}\left(\bar{v}_{j}\right)\right]_{j=J_{1}}^{J_{2}},\left[\triangle w_{o H, t+1}\left(\bar{v}_{j}\right)\right]_{j=J_{3}}^{J_{4}}\right\} \\
y_{t}^{2}=\left\{\triangle u_{y L, t}, \triangle u_{y H, t} \triangle n_{y L, t}, \triangle n_{y H, t} \triangle \ln \xi_{y t}, \triangle \ln \lambda_{t}, \triangle \ln q_{t},\left[\triangle \ln \eta_{t}\left(\bar{v}_{j}\right)\right]_{j=1}^{J},\right. \\
{\left[\triangle u_{o L, t}\left(\bar{v}_{j}\right)\right]_{j=1}^{J},\left[\triangle u_{o H, t}\left(\bar{v}_{j}\right)\right]_{j=1}^{J},\left[\triangle n_{o L, t}\left(\bar{v}_{j}\right)\right]_{j=1}^{J},\left[\triangle n_{o H, t}\left(\bar{v}_{j}\right)\right]_{j=1}^{J},} \\
\left.\left[\triangle \ln \xi_{o t}\left(\bar{v}_{j}\right)\right]_{j=1}^{J}, \triangle \ln H_{t}, \triangle I_{t}\right\}
\end{gathered}
$$

The following Table classifies the different equations into five types. Types 1-5 correspond to equations (2.23)-(2.27), respectively. 


\begin{tabular}{|c|c|c|c|}
\hline Equation \# & Equation name & \# of equations & Type $(1,2,3,4$ or 5$)$ \\
\hline 1 & $L_{u_{y L}}$ & 1 & 5 \\
\hline 2 & $L_{u_{y H}}$ & 1 & 5 \\
\hline 3 & $L_{n_{y L}}$ & 1 & 5 \\
\hline 4 & $L_{n_{y H}}$ & 1 & 5 \\
\hline 5 & $L_{w_{y L}}$ & 1 & 4 \\
\hline 6 & $L_{w_{y H}}$ & 1 & 4 \\
\hline 7 & $L_{u_{o L}\left(\bar{v}_{i}\right)}$ & $J$ & 5 \\
\hline 8 & $L_{u_{o H}\left(\bar{v}_{i}\right)}$ & $J$ & 5 \\
\hline 9 & $L_{n_{o L}\left(\bar{v}_{i}\right)}$ & $J$ & 5 \\
\hline 10 & $L_{n_{o H}\left(\bar{v}_{i}\right)}$ & $J$ & 5 \\
\hline 11 & $L_{w_{o L}\left(\bar{v}_{i}\right)}$ & $J_{2}-J_{1}+1$ & 4 \\
\hline 12 & $L_{w_{o H}\left(\bar{v}_{i}\right)}$ & $J_{4}-J_{3}+1$ & 4 \\
\hline 13 & $L_{I C^{y}}$ & 1 & 5 \\
\hline 14 & $L_{I C^{o}\left(\bar{v}_{i}\right)}$ & $J$ & 5 \\
\hline 15 & $L_{P K^{o}\left(\bar{v}_{i}\right)}$ & $J$ & 5 \\
\hline 16 & $L_{q}$ & 1 & 5 \\
\hline 17 & $L_{\lambda}$ & 1 & 5 \\
\hline 18 & $L_{Y}$ & 1 & 1 \\
\hline 19 & $L_{H}$ & 1 & 1 \\
\hline 20 & $L_{I}$ & 1 & 3 \\
\hline 21 & $L_{\mu}$ & $(N+1)\left[2+\left(J_{2}-J_{1}+1\right)+\left(J_{4}-J_{3}+1\right)\right]$ & 2 \\
\hline
\end{tabular}

Total number of equations:

$$
12+6 J+\left(J_{2}-J_{1}+1\right)+\left(J_{4}-J_{3}+1\right)+(N+1)\left[2+\left(J_{2}-J_{1}+1\right)+\left(J_{4}-J_{3}+1\right)\right]
$$

Total number of Type 1 equations: 2

Total number of Type 2 equations: $(N+1)\left[2+\left(J_{2}-J_{1}+1\right)+\left(J_{4}-J_{3}+1\right)\right]$

Total number of Type 3 equations: 1

Total number of Type 4 equations: $2+\left(J_{2}-J_{1}+1\right)+\left(J_{4}-J_{3}+1\right)$

Total number of Type 5 equations: $7+6 J$ 
The following is the dimensionality of the different variables:

$$
\begin{gathered}
\operatorname{dim}\left(x_{t-1}^{1}\right)=(N+1)\left[2+\left(J_{2}-J_{1}+1\right)+\left(J_{4}-J_{3}+1\right)\right] \\
\operatorname{dim}\left(x_{t-1}^{2}\right)=1 \\
\operatorname{dim}\left(y_{t}^{1}\right)=2+\left(J_{2}-J_{1}+1\right)+\left(J_{4}-J_{3}+1\right) \\
\operatorname{dim}\left(y_{1}^{2}\right)=9+6 J \\
\operatorname{dim}\left(z_{t}\right)=1
\end{gathered}
$$

Total number of endogenous variables: $(N+1)\left[2+\left(J_{2}-J_{1}+1\right)+\left(J_{4}-J_{3}+1\right)\right]+12+\left(J_{2}-J_{1}+1\right)+$ $\left(J_{4}-J_{3}+1\right)+6 J$ (same as number of equations).

\subsection{Simplified linear system}

Under the classifications of the previous section, the linearized model given by equations (2.23)(2.28) simplifies to the following:

$$
\begin{aligned}
0 & =B_{11} x_{t}^{1}+B_{12} x_{t-1}^{2}+C_{12} y_{t}^{2}+D_{1} z_{t} \\
0 & =A_{21} x_{t+1}^{1}+B_{21} x_{t}^{1}+C_{21} y_{t+1}^{1} \\
0 & =A_{32} x_{t}^{2}+B_{32} x_{t-1}^{2}+C_{32} y_{t}^{2} \\
0 & =J_{42} y_{t+1}^{2}+K_{41} y_{t+1}^{1}+K_{42} y_{t}^{2} \\
0 & =E_{t}\left\{G_{52} x_{t}^{2}+H_{52} x_{t-1}^{2}+J_{52} y_{t+1}^{2}+K_{51} y_{t+1}^{1}+K_{52} y_{t}^{2}+L_{5} z_{t+1}+M_{5} z_{t}\right\}
\end{aligned}
$$

and the transformation given by equations (2.37)-(2.41) simplifies to:

$$
\begin{aligned}
\Theta_{1} & =\Upsilon A_{21}^{-1} C_{21} K_{41}^{-1} J_{42} S_{2}, \\
\Psi_{1} & =\Upsilon\left[A_{21}^{-1} C_{21} K_{41}^{-1} J_{42} R_{22} Q_{2}+A_{21}^{-1} C_{21} K_{41}^{-1} K_{42} S_{2}\right], \\
\Lambda_{1} & =-K_{41}^{-1} J_{42} R_{21} \Theta_{1}-K_{41}^{-1} J_{42} S_{2}, \\
\Gamma_{1} & =-K_{41}^{-1} J_{42} R_{21} \Psi_{1}-K_{41}^{-1} J_{42} R_{22} Q_{2}-K_{41}^{-1} K_{42} S_{2},
\end{aligned}
$$

where

$$
\Upsilon=\left[I-A_{21}^{-1} C_{21} K_{41}^{-1} J_{42} R_{21}\right]^{-1}
$$

